\title{
INVESTIGATION OF THE BEHAVIOR OF FREE-STANDING GABIONS \\ IN SEISMIC REGIONS
}

\author{
A Thesis \\ presented to \\ the Faculty of California Polytechnic State University \\ San Luis Obispo, California
}

In Partial Fulfillment

of the Requirements for the Degree

Master of Science in Architecture with a Specialization in Architectural Engineering

by

Amy Ransom

March 2011 
(C) 2011

Amy Ransom

ALL RIGHTS RESERVED

ii 
COMMITTEE MEMBERSHIP

TITLE:

INVESTIGATION OF THE BEHAVIOR OF FREE-STANDING GABIONS IN SEISMIC REGIONS

AUTHOR:

AMY RANSOM

DATE SUBMITTED:

MARCH 16, 2011

COMMITTEE CHAIR: Abraham Lynn, PhD.

COMMITEE MEMBER: Graham Archer, PhD., PEng.

COMMITTEE MEMBER: Kevin Dong, S.E. 


\section{ABSTRACT \\ Investigation of the Behavior of \\ Free-Standing Gabion Walls in Seismic Regions \\ Amy Ransom}

This thesis investigates the behavior of free-standing gabion walls in areas of mild seismicity. To investigate this behavior, three walls of varying internal cable patterns were constructed at a quarter-scale. These walls were tested with a mass shaker, and mode shapes, tone extractions, and damping ratios were calculated. A modal analysis was conducted using design spectra created from a suite of seven earthquakes from countries bordering Kenya. The corresponding lateral forces to these spectral displacements were found, and the restoring eccentricities from the soil restoring force were backed out through a summation of moments. All analysis was experimentally done due to the complexity of properly modeling the wall system for a secondary analytical comparison.

This process was done under the assumption of linear behavior. Similarly, the criteria for failure involved the eccentricity of the restoring soil force exceeding the kern distance (assuming elastic behavior)—criteria that all three of the wall specimens met. However, further research into the nonlinear behavior of this wall type is suggested for future conclusions on free-standing gabion wall behavior.

Keywords: Gabion, Free-standing, Seismic, Africa, Kenya 


\section{ACKNOWLEDGEMENTS}

A thanks to those who helped me along this journey and made this thesis possible:

My parents, for supporting me through this process and believing in me, despite all the twists and turns in procedures and results. This thesis is not just my victory, but ours.

My friends, who still considered themselves such when I disappeared for days and weeks on end working on this thesis.

Central Coast Steel Fabricators, for donating part of the materials that made this project possible at all.

Wayne Montgomery, for the additional assistance in research and assurance that this thesis was indeed necessary.

Ray Ward, for all the continuous patience and assistance in everything labrelated. Your knowledge and expertise with the lab equipment made this experiment possible, and I could not have done it without you.

My main advisors, Drs. Abe Lynn and Graham Archer, for your immeasurable support and guidance in this process. Truly, there are no words for how vital you are.

Most importantly, my Lord and Savior Jesus Christ, Who faithfully guided my path in this process, and gave me the strength and endurance to complete this thesis and serve Him in this way. 


\section{TABLE OF CONTENTS}

Page

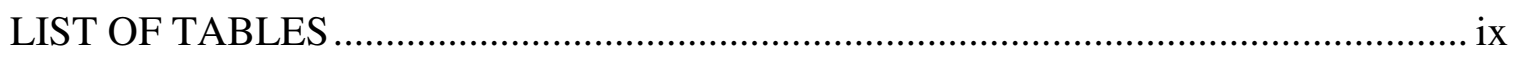

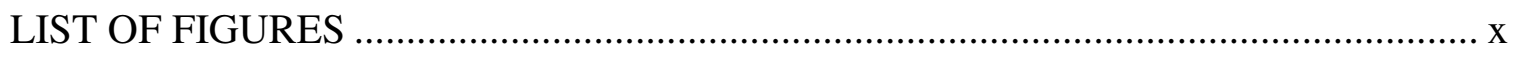

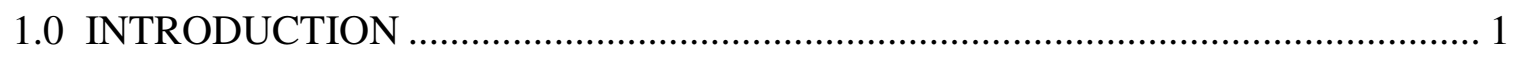

1.1 Purpose

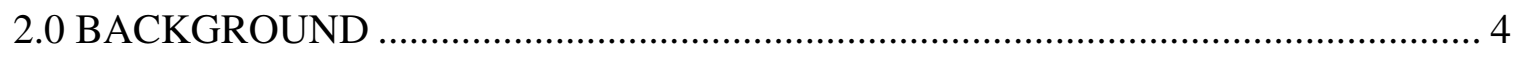

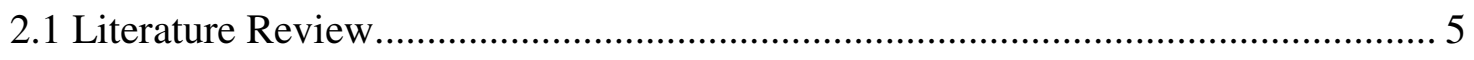

3.0 EXPERIMENTAL SET-UP AND TESTING EQUIPMENT ……............................. 7

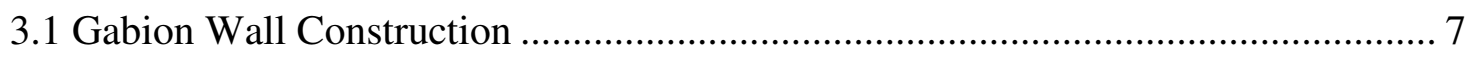

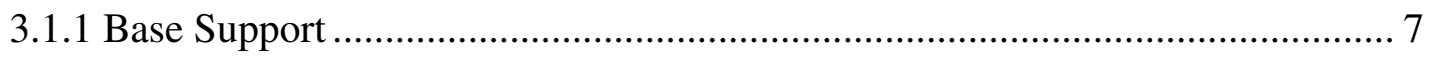

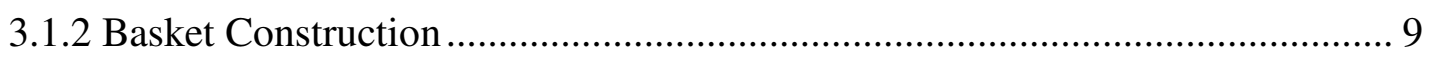

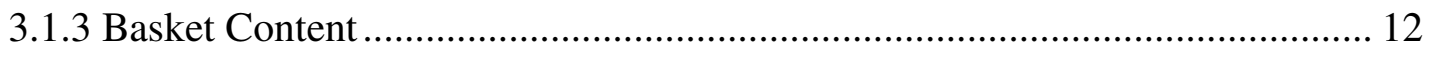

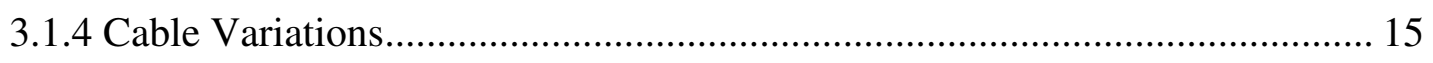

3.2 Forced Vibration Test Equipment.................................................................. 18

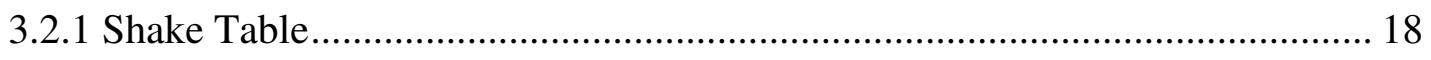

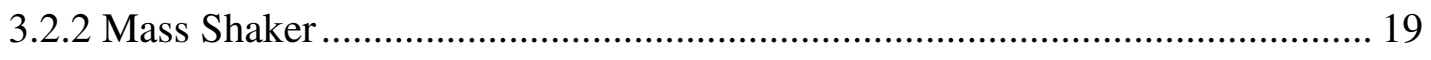

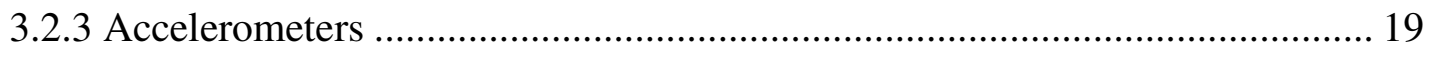




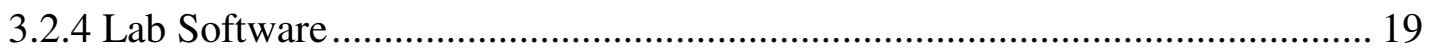

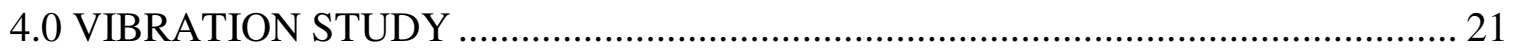

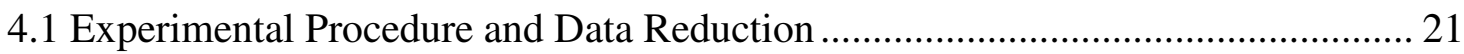

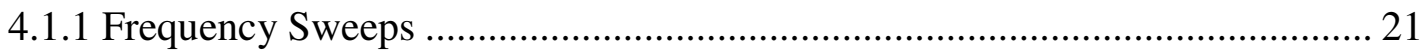

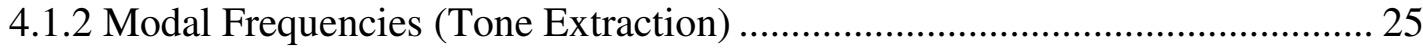

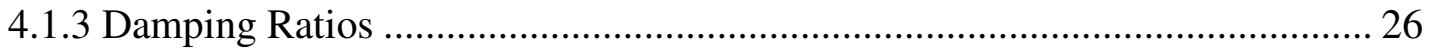

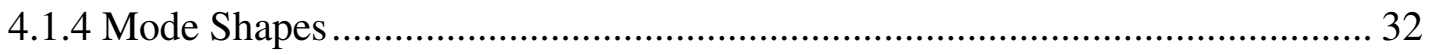

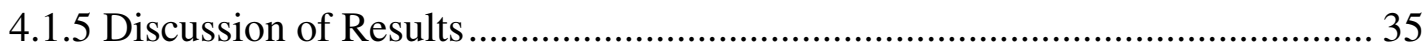

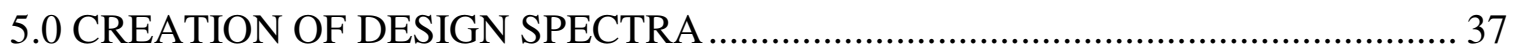

5.1 Explanation of Design Spectra.............................................................................. 37

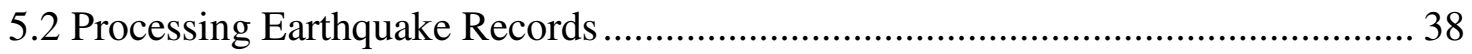

5.3 Determination of Spectrum Coefficients ................................................................ 40

5.4 Creation of Mode-Specific Design Spectra ………............................................ 42

5.4.1 Creating the Constant Spectrum Regions ...................................................... 43

5.4.2 Creating the Amplified Spectrum Regions ....................................................... 44

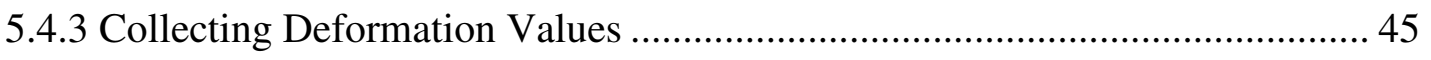

6.0 ANALYSIS OF VIBRATION PROPERTIES …………........................................ 47

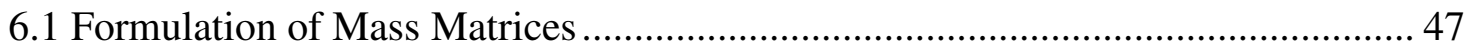




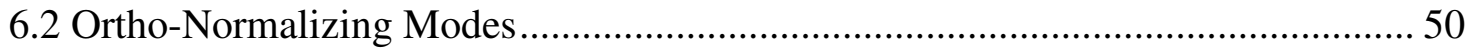

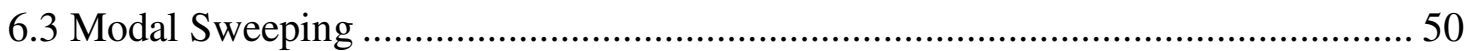

6.4 Mass Participation Factors .............................................................................. 52

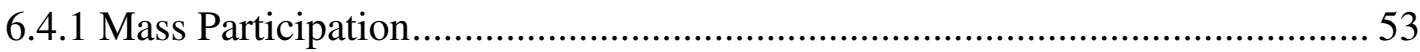

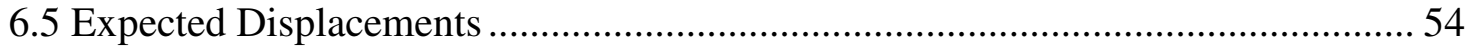

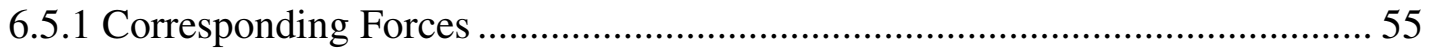

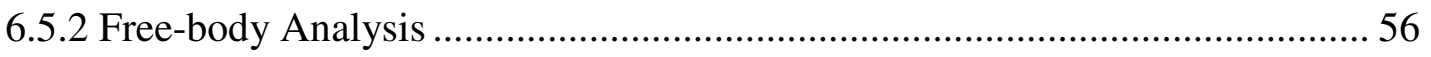

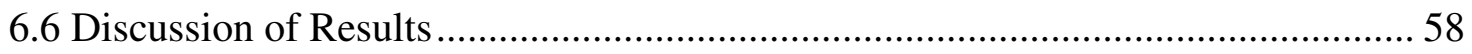

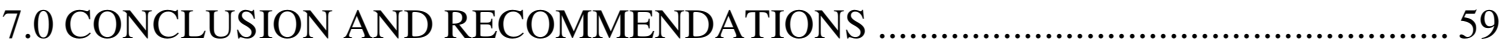

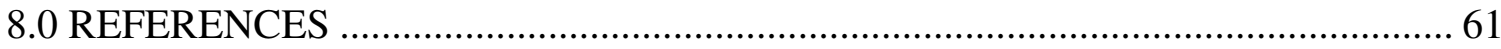

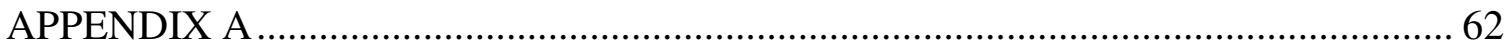

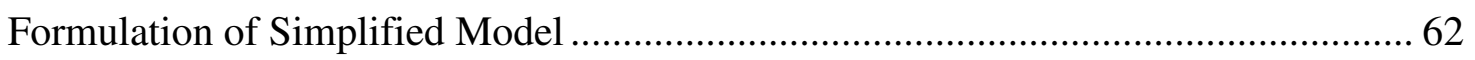

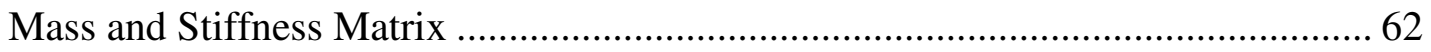

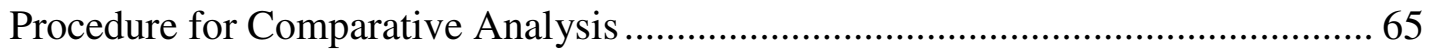

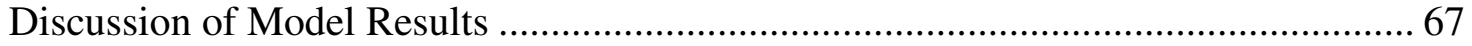




\section{LIST OF TABLES}

Page

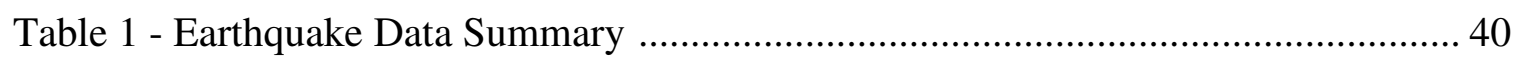

Table 2 - Spectrum Amplification Factors ............................................................... 41

Table 3 - Amplification Factor Summary ............................................................. 42

Table 4 - Amplified Spectrum Results.............................................................. 42

Table 5 - Summary of Wall Data for Spectrum Creation .......................................... 43

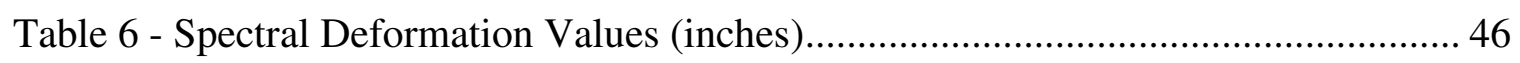

Table 7 - Summary of level displacements for each wall ........................................... 55

Table 8 - Summary of forces for each wall (lbs.) ….............................................. 56

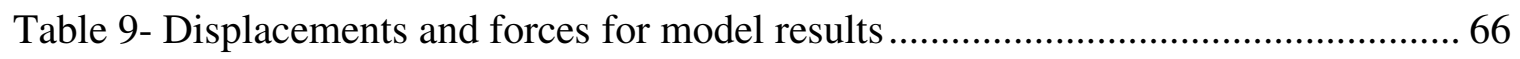




\section{LIST OF FIGURES}

Page

Figure A - Gabions used as a retaining wall ............................................................. 4

Figure B - Gabions used for 16th-Century artillery protection........................................... 4

Figure C - Cross Section of Constructed Wall ........................................................... 7

Figure D - Shear vs. Flexural Behavior and Base Interaction........................................... 8

Figure E - Cross Section of Wall Base ...................................................................... 9

Figure F - Gabion Construction Detail ................................................................. 10

Figure G - Basket frame layout.............................................................................. 10

Figure H - Proposed wall elevation ......................................................................... 11

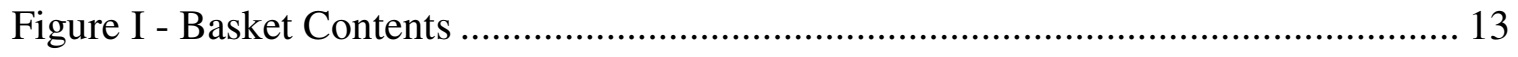

Figure J - Basket content elevation ........................................................................... 14

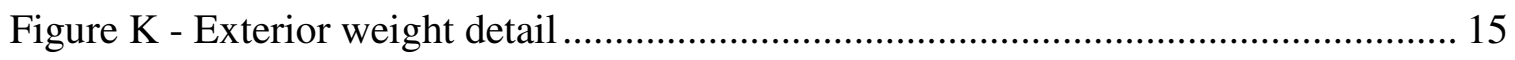

Figure L - Basket Content between constructions .......................................................... 15

Figure M - Wiring detail .................................................................................... 15

Figure N - In-progress wall construction ............................................................. 16

Figure $\mathrm{O}$ - Variations in Wiring Details..................................................................... 17

Figure P - Disconnected hydraulic pump................................................................ 18

Figure Q - Testing equipment ......................................................................... 20

Figure R - Lab software output from Wall A Testing ……………................................. 22

Figure S - Superimposed frequency sweep for Wall A .................................................. 24 
Figure T - Tone Extraction for Wall C

Figure U - Half-Power Bandwidth Process ................................................................ 27

Figure V - "Half Half-Power Bandwidth" Process ………….......................................... 28

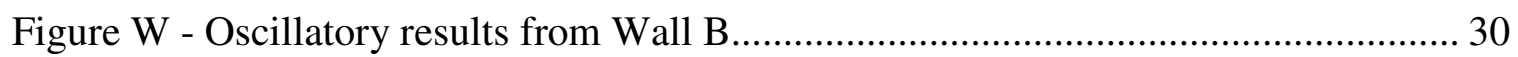

Figure X - Damping through oscillatory decay measurement .......................................... 31

Figure Y - Apparent First and Second Mode Shapes..................................................... 33

Figure Z - Constructed Wall Elevation ....................................................................... 33

Figure AA - Lab results for synchronizing check....................................................... 35

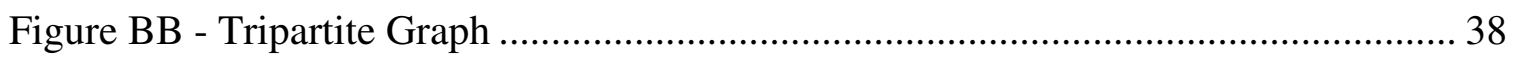

Figure CC - Excerpt from map of Africa …………………........................................ 39

Figure DD - Beginning stages of design spectrum creation ............................................ 44

Figure EE - Design Spectrum with amplified regions .................................................. 45

Figure FF - Design Spectrum with measured Deformation values.................................... 46

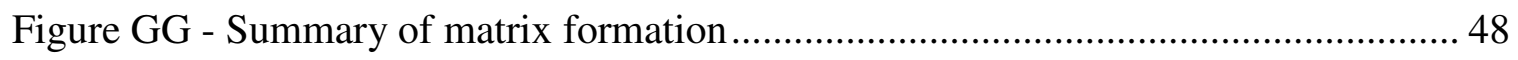

Figure HH - Shape Functions for Wall ........................................................................ 49

Figure II - Free body diagram for summation of moments ............................................. 57 


\subsection{INTRODUCTION}

This thesis investigates using gabion wall units for potential residences. A simple definition for a gabion wall unit would be a wire box filled with rocks used for structures such as retaining walls (which generally hold back earth or help maintain other potential landslide areas). A further explanation and images of gabions may be found in the Background (Section 2.0) portion of this thesis.

The Purpose (Section 1.1) presents the main motive behind this project: investigating the behavior of free-standing gabion walls, with specific focus on out-ofplane behavior. Also, this section will explore the intended experiments to be paired with this thesis.

\subsection{Purpose}

The purpose of the project is to investigate out-of-plane gabion behavior for freestanding systems in seismic areas. A main goal of the project is to add the findings of this thesis to the data base of existing gabion research due to the lack of information currently available. A secondary intention of this thesis would be to have this idea further researched to find a potential low-cost housing construction technique, especially for developing nations in need of such a construction option.

The experiment consists of constructing a scaled, single wall line (versus the entire housing unit) in the same method that a larger wall would be constructed. The small scale not only allows compatibility with shake table dimension restrictions, but also permits the construction of multiple wall lines; more specifically, testing the performance 
of varying tie-down designs. The wall line will be fastened to and tested by a shake table after construction to ensure the seismic integrity of the gabion wall design. If the multiple tests prove the design to be adequate per success criterion, the wall configuration would then be considered a viable construction option. The criterion for success is twofold: experimental and analytical. Experimentally, failure will have occurred if the freestanding wall design does not remain standing under experimental shaking. Analytically, failure will have occurred if the forces derived from the modal analysis show that the system to be unable to withstand seismic influence.

The constructed model wall is a scaled version of what the actual wall should be dimensioned to. Concrete (or comparable) systems can be scaled to a minimum of $25 \%$ the original size (Zarnic, et al. 2001) while maintaining accurate results. This limit is because of the restrictions in aggregate performance: after a certain size, the aggregate performance (and corresponding results) cannot accurately be compared to the largerscaled version.

As with any concrete construction project, tie-downs must be involved in construction to ensure that all units in construction are integrated. In masonry construction, this integration is the mortar between the bricks and the reinforcement threaded vertically through the concrete masonry units. With gabions (as discussed in the Background (Section 2.0) portion of this thesis), the binding element is the organic material allowed to filter through the units, which acts as a cement over time. For this 
project, a series of cables will be used to "sew" the blocks together to imitate this necessary bonding (Simac, et al. 1997). 


\subsection{BACKGROUND}

Though currently used primarily in the construction industry throughout the world, the gabion has been around for centuries. The word "gabion" was derived from the Italian word gabbione, meaning "big cage". Originally invented by Leonardo da Vinci, the gabion was created as a support system for the castle San Marco in Milan (Felix and Germain, 1996).

Later, the gabion spread to military use and transformed into a wicker structure. These wicker

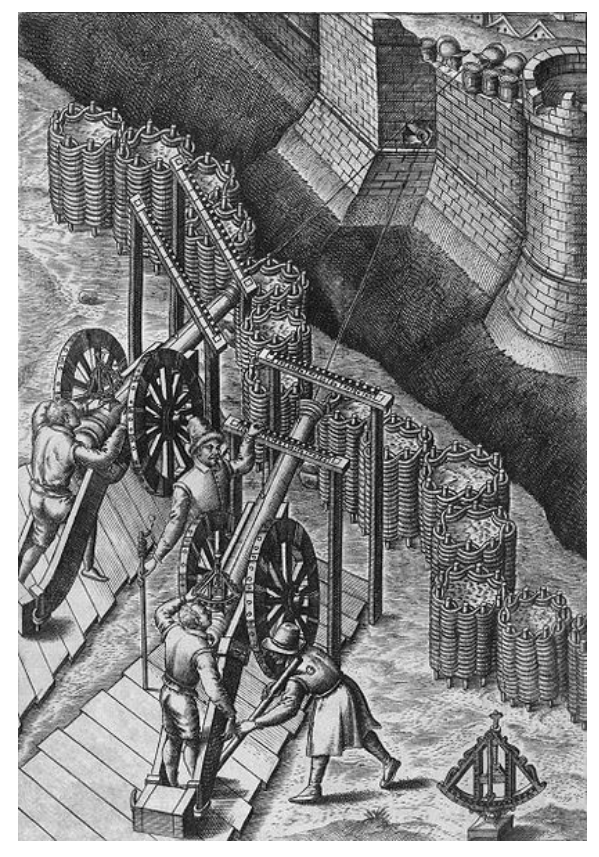

Figure B - Gabions used for 16th-Century artillery protection http://gabiondesign.be/gabion.html shells were transported empty during battle, then staked into place and filled with soil to protect the artillery gunners (see Fig. B above).

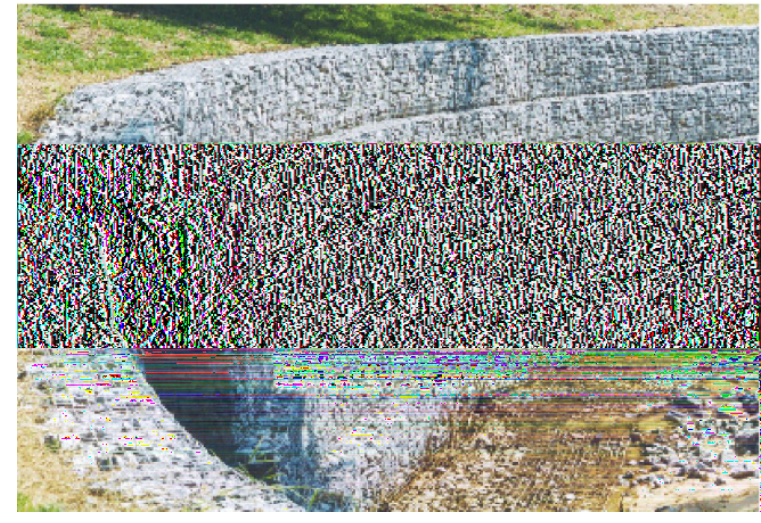

Figure A - Gabions used as a retaining wall to traffic areas and waterways. The gabion

They had no top or bottom and were made in different diameters to allow layering during transportation.

Currently, the gabion wall is most commonly used in civil engineering applications, as it currently applies mostly wall unit is used mostly for shore stabilization against erosion. Other uses include 
retaining walls, temporary floodwalls, silt filtering, semi-permanent dams, or even a fish barrier. Gabion walls can become more efficient with time when used as retaining structures. This happens because silt and vegetation can fill the voids between the rocks, sometimes creating higher strength and better retaining effectiveness (Shevchenko, 1996).

Gabions are looked upon favorably as retaining units for many reasons. First, their modularity allows for them to be stacked in various shapes, conforming to the demands of the site. Second, their lack of rigidity as an entire structure allows for continuous conformation to potential ground movement (for example, settlement or expansion). This lack of rigidity means that portions of the stacked units can shift with the earth surrounding it without compromising the structural system of the neighboring gabion units. In relation to water systems, gabion units can dissipate energy from flowing water and allow proper drainage (Shevchenko, 1996).

\subsection{Literature Review}

Gabion walls have been a civil engineer's building element for many years. Within their primary use as erosion-prevention systems, dozens of papers and experiments have investigated the behavior of and possible improvements to the gabion wall system. Some of these investigations even include seismic behaviors-but all within the realm of use as a retaining system.

Though gabions have not been investigated as a construction option, the use of adobe brick product has been. Adobe is used because it requires unskilled labor for FREE-STANDING GABIONS IN SEISMIC REGIONS 
construction, and utilizes materials readily available in the immediate area, either naturally-occurring (sand), or by purchase (cement), though these purchased materials are very costly. However, this system has proven to be susceptible to moisture, making adobe a poor choice in the search for a system with greater longevity through rainy seasons (Chen, 2009). Gabion walls also present an initial investment for materials like wire mesh, but have the capacity to last through multiple rainy seasons with minimal repairs, ideally.

This thesis investigates the possibility of a free-standing gabion wall system with potential application to construction options. In researching literature dealing specifically with free-standing gabion wall systems, nothing appears to be in publication. The purpose of this thesis, therefore, begins to fill in the voids in understanding free-standing gabion wall behavior, more specifically, walls located in areas of mild seismicity. 


\subsection{EXPERIMENTAL SET-UP AND TESTING EQUIPMENT}

This section explains the experimental set-up, including construction of the gabion walls and the variations between tests, as well as equipment used in the forcedvibration testing.

\subsection{Gabion Wall Construction}

Gabion wall construction is broken into three parts: the base support (which includes the shake table), basket construction, and the various cable patterns for each wall type.

\subsubsection{Base Support}

The base support is an aspect of testing that went through many designs before it was actually implemented. The initial idea behind having a support at all is to try to re-create the effect of having a partially-embedded wall. By creating a base that is offset from the wall, soil can be filled in between the wall and the supports to re-create the lateral soil support that a real wall would experience (see Figure $\mathrm{C}$ below).

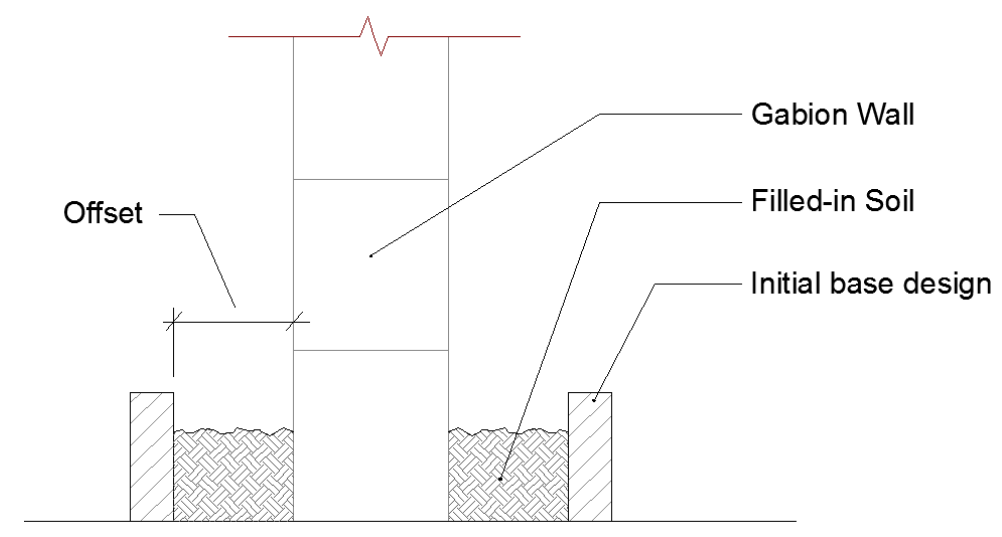

Figure C-Cross Section of Constructed Wall

FREE-STANDING GABIONS IN SEISMIC REGIONS 
The problem with this initial design became how to accurately re-create the soil response. One issue came purely from a constructability standpoint: the wall design took up the entire four-foot width of the four-feet-wide, eight-feet-long shake table, so having an exterior base support create additional width to the design was difficult. The second issue was from a technical standpoint: in creating any system that involved soil response, accuracy became a major concern. If the support is too close to the wall, then the soil will have an artificially high stiffness. However, the physical dimensions of the shake table prohibited a support system far enough away from the wall to create accurate soil behavior.

The second design issue was how high the support should be. Since free-standing gabion wall behavior is relatively un-documented, there was no way to know whether the performance will be shear- or flexure-dominated. If shear, a higher base might interfere with the natural behavior (Fig. D, left), while this base height would not be so much of a concern for flexural behavior (Fig. D, right).

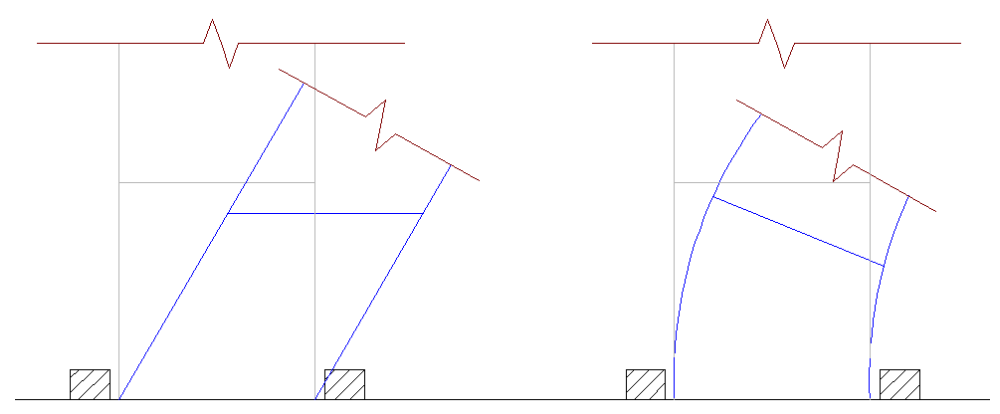

Figure D - Shear vs. Flexural Behavior and Base Interaction (left and right, respectively)

FREE-STANDING GABIONS IN SEISMIC REGIONS 
In the end, the base support was used more for displacement resistance than it was for soil response. A small running block was built along the perimeter at an approximate $1 / 8^{\prime \prime}$ offset from the wall base to prohibit any large lateral displacements (see Figure E below). This allows un-inhibited rocking, should rocking occur, but also limits potential large displacements.

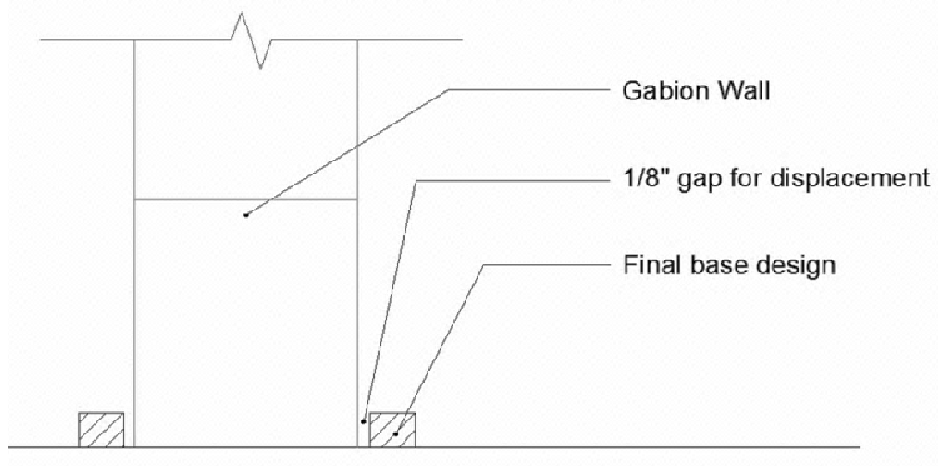

Figure E-Cross Section of Wall Base

\subsubsection{Basket Construction}

The baskets themselves are designed as closely to actual gabion baskets as possible, though they are one-quarter scale. The specific design is modeled after the one shown on the following page (see Figure F) (MGS, 2006). 


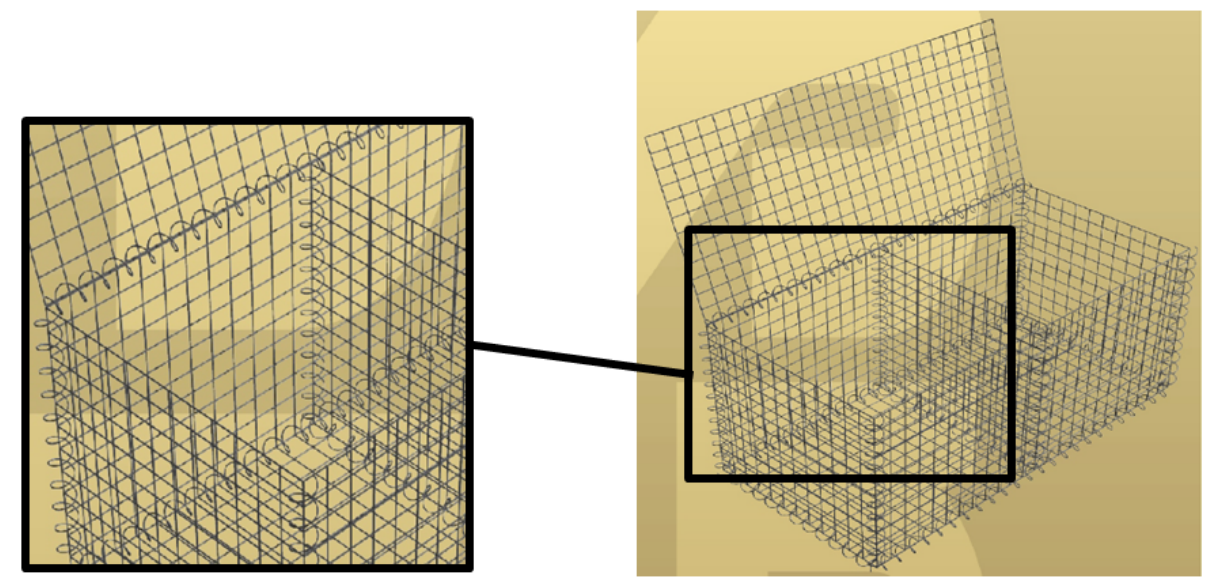

Figure F - Gabion Construction Detail

The basket wall is cut from 16 gauge $1 / 4^{\prime \prime}$ square wire mesh, then bent to form (see Figure $\mathrm{G}$ below) and wired as seen in the close-up of Figure F. The center membrane piece is cut separately and wired in place at the mid-point of the block dimension to minimize out-of-plane bowing of the longer basket walls, also seen in Figure F above.
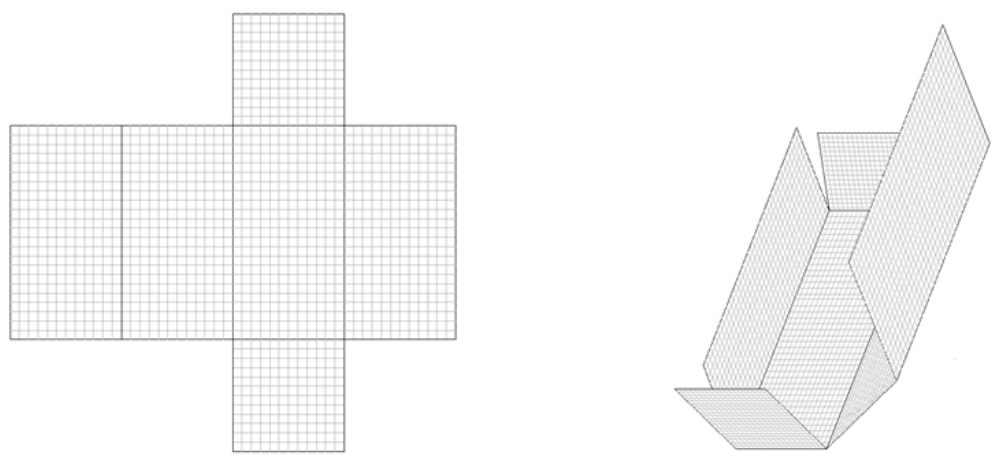

Figure G - Basket frame layout

The desired wall design replicates a wall with the following dimensions: $2^{\prime}$ in wall thickness, $8^{\prime}$ in wall height, and an undetermined length. This creates a 1:4 ratio in wall 
thickness to height. The blocks themselves generally have a 1:2 height to length ratio; an $8^{\prime}$ wall requires a minimum base length of $16^{\prime}$ to accurately represent the wall design. However, the shake table supports only a 4' length, requiring the wall to be scaled down to $25 \%$ of its actual size. This percentage is also the limit on scaling concrete (or similar) models down before the interaction of the aggregate becomes inaccurate (Zarnic, et al. 2001) .

An available length of $4^{\prime}$ results in a $2^{\prime}$ height per the 1:2 aspect ratio. A single column of baskets would not capture the interaction the baskets would experience as part of a wall unit, so a "pyramid" design is required to more accurately capture load flow and behavior. For a "pyramid" design, the wall needs 4 blocks on the bottom row, 3 on the next, then 2, and then 1 block on top, as seen in Figure H below.

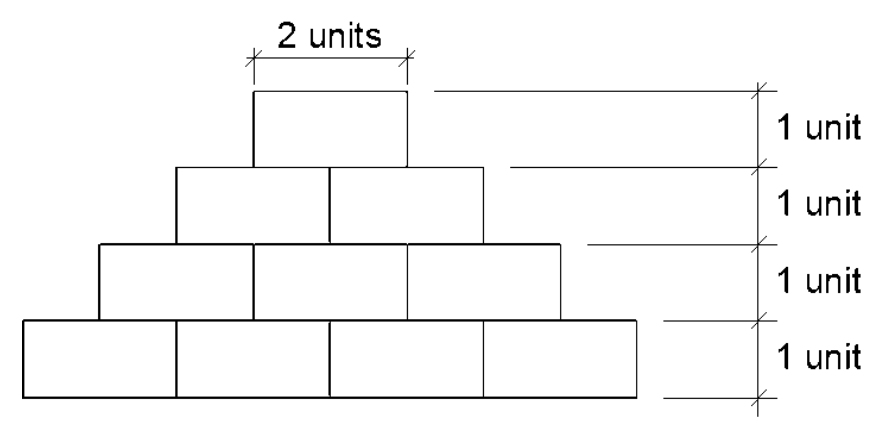

Figure H - Proposed wall elevation

With four blocks covering 4' of length, the blocks need a unit length of 1'. Thus, with the 1:2 height-to-length ratio, each of the blocks will need to have a 6 " height and width . 


\subsubsection{Basket Content}

Ideally, the baskets would have been filled solely with rocks after being wired to form. However, as previously mentioned, the shake table dimensions limit the length of the wall, resulting in scaling the wall down to $25 \%$ of full size. The actual basket contents will be expanded on in Basket Content Analysis (Section 3.1.3.2). A process called "similitude" is required to maintain accurate results when scaling a model down.

\subsubsection{Similitude}

The basic idea of similitude is that, in scaling the model, certain parameters of the experiment are adjusted proportionally to deliver accurate results when tested.

In every model, certain properties cannot easily be changed, like gravity or time. But things that can be changed include properties like length, mass, and force. "The Buckingham Pi” theorem in Fluid Dynamics investigates this specific issue of similitude (Buckingham, 1915). Through the process outlined in the theorem, variables within the experiment are selected and processed to keep consistent results between full-size and model-sized experiments.

For this specific experiment, the length and mass are the basic variables chosen to maintain experimental accuracy. The two turn out to be inversely proportional, so reducing the "length" of the model 4 times results in a 4-fold required increase in the "mass." 


\subsubsection{Content Analysis}

In order to find the mass required, a single basket full of typical aggregate from the ARCE concrete lab on campus is weighed. This basket full weighed approximately 20 pounds, so four times that weight was approximately 79 pounds per block.

A basket volume of $0.25 \mathrm{ft}^{3}$ requires material with a

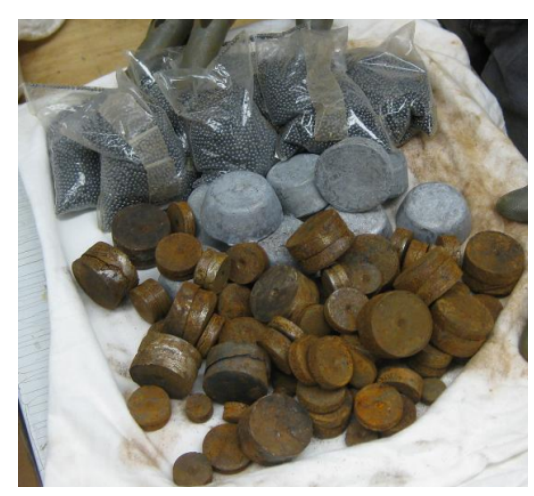

Figure I - Basket Contents minimum density of $320 \mathrm{lb} / \mathrm{ft}^{3}$ if the walls are to be filled completely by one material. The aggregate from the concrete lab has a measured density of $95 \mathrm{lb} / \mathrm{ft}^{3}$, so this material is not acceptable for use on its own. Steel has a significantly higher density than aggregate, so donated steel plugs left over from dye punches aided greatly in construction. The plugs have a measured density of $446 \mathrm{lb} / \mathrm{ft}^{3}$, though only 147 pounds of the material is available.

Using lead in conjunction with the aggregate and steel pieces reached the required wall weight and minimized the addition of exterior weights. Lead has a density of 709 $\mathrm{lb} / \mathrm{ft}^{3}$ and would be ideal for mixing with the aggregate to reach the required wall weight of approximately $800 \mathrm{lbs}$. Just over 750 pounds of lead and other steel material was available for use. The amount of lead was significantly less than was required to contain the entirety of the wall weight within the baskets. This meant that exterior weights are 
required, though they will need to hang as close as possible to the structure to minimize any influence on the structure's behavior.

\subsubsection{Final Content Design}

In the end, it was decided that the center baskets in the wall should remain as true to ideal design as possible (see Figure $\mathrm{J}$ below). The idea behind this restriction is to allow the wall to perform as naturally as possible without exterior influence. In application, this means that the center blocks in the wall will consist of the lead/steel/aggregate mixture with no exterior weights. The outer blocks will consist mainly of steel and aggregate and also support the exterior weights required per the similitude analysis. These weights will be placed as low as possible on the structure to minimize any potential influence in regard to displacements and dynamic behavior. Figures $\mathbf{J}$ (below) and $\mathrm{K}$ (next page) show the final wall design in regard to dispersion of weight among the blocks.

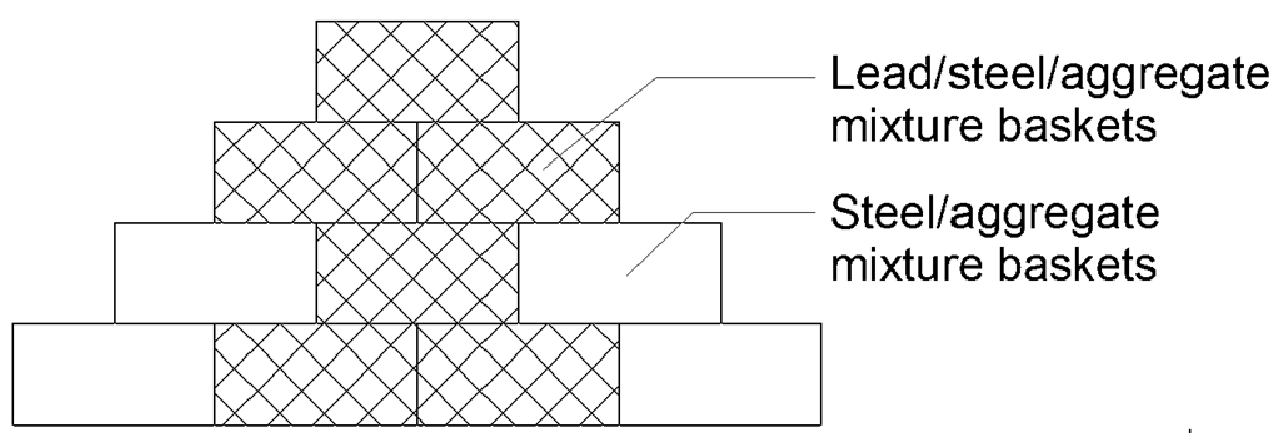

Figure J - Basket content elevation 


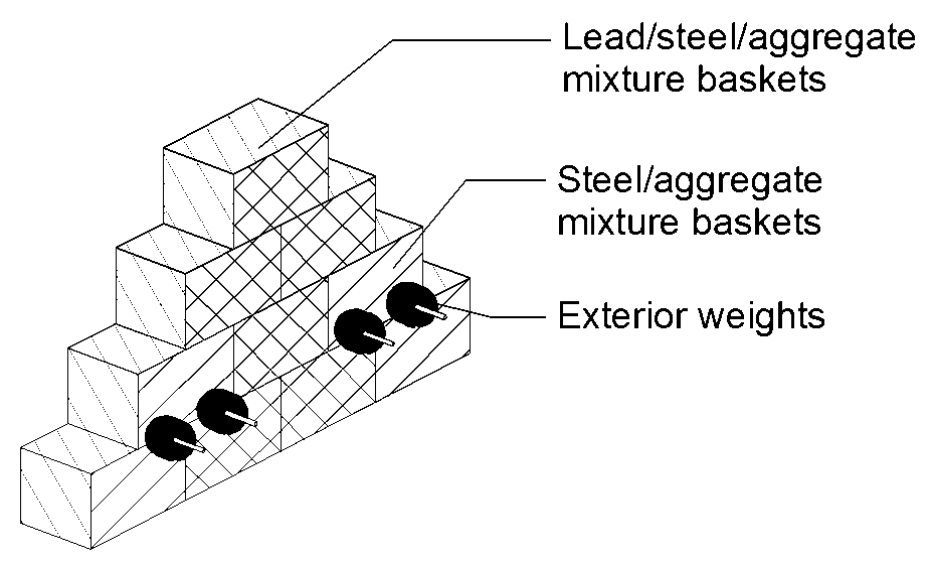

Figure $K$ - Exterior weight detail

\subsubsection{Cable Variations}

The biggest variation between the three wall types to be tested is the cable design for each wall. The filling remains the same, and is kept separate between wall builds as seen below in Figure L. The cables are laced through the baskets in three different patterns, as seen in page 17. The cables are hand-tied to the edges of the basket using 20guage wire. This tie occurs at any point where a 90-degree bend in the cable is necessary, or where close contact with the basket was vital. The connection was vital

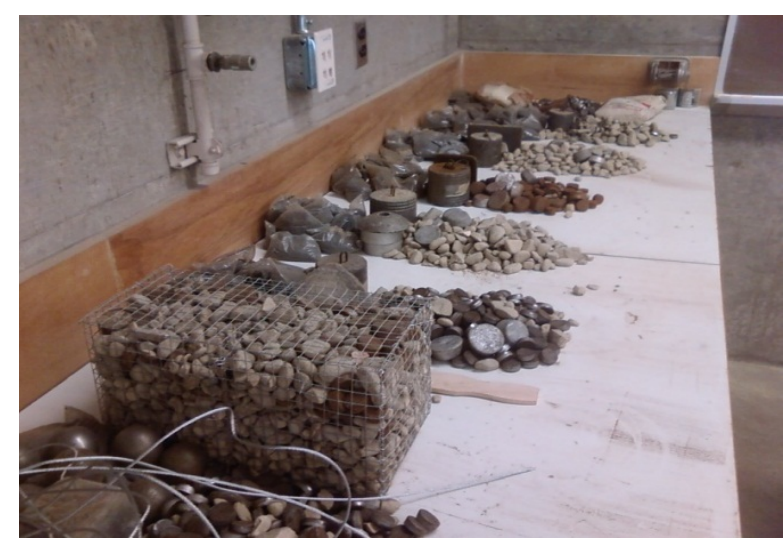

isket Content nstructions

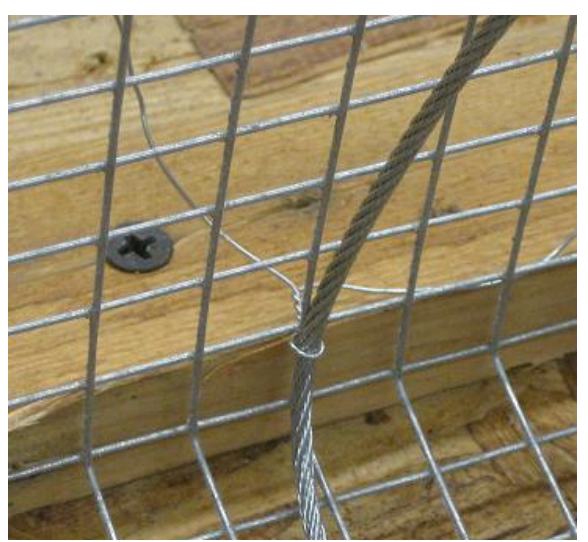

ing detail

ISMIC REGIONS 
because the cables, unrestrained, could add artificial flexibility to the wall. The image for that bend detail can be seen above in Figure M. An in-progress construction shot can be seen in Figure $\mathrm{N}$ below as well.

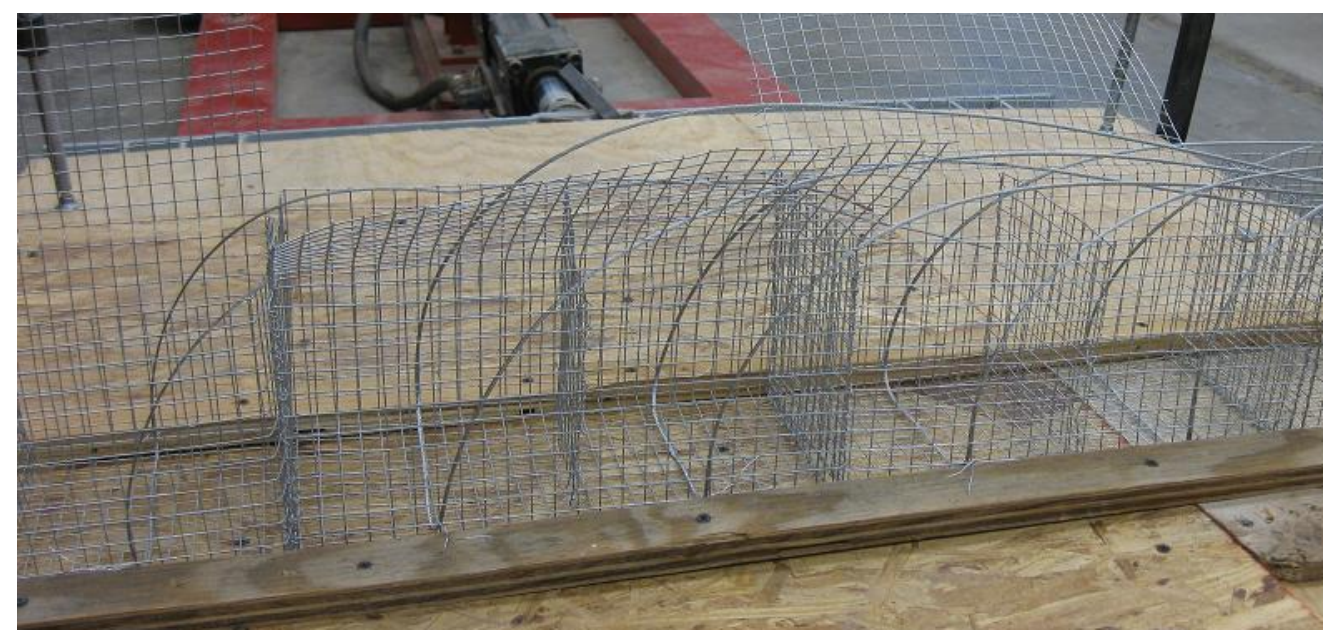

Figure N - In-progress wall construction

Another concern in designing the walls was how to connect the accelerometers needed to measure different wall behaviors (see Section 3.2.3). Thin wooden paddles were placed between each layer as the wall was being built. These paddles protrude far enough from the structure to mount the accelerometers, but are close enough to gather accurate data when the dynamic analysis was underway since measurement from inside the wall was not an option.

As seen in Figure $\mathrm{O}$ on the following page, there are three types of cable designs for the different walls. The cables are shown in blue, while the hand-tie wire pieces are represented by the red segments. Initially, the idea was to build the same wall design three times to gather consistent results. However, after being unable to find any research 
on this specific topic of gabion wall study, the focus changed. Rather than hone in on one specific cable pattern and repeat the experiment three times, the focus changed to altering the cable patterns and finding the most successful pattern as a basis for future research.

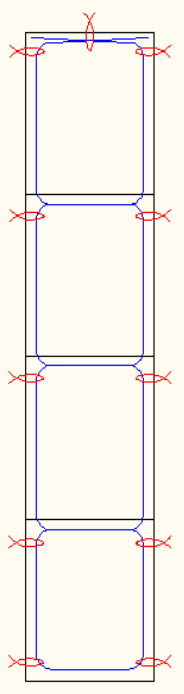

(A)
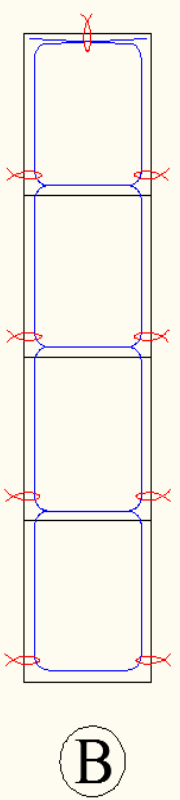

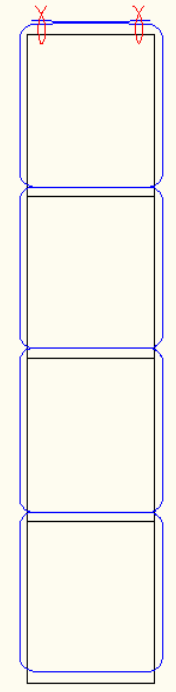

(C)

Figure $O$ - Variations in

Wiring Details

The first wall, labeled " $A$ " in Figure $\mathrm{O}$, has cables that overlapped through a basket, top and bottom, before entering the next basket. The idea behind this design is to minimize rocking and lift-up action between the baskets by enclosing the upper cable overlap entirely in one basket. This wall is denoted by reference to "Wall A".

The second wall, labeled " $\mathrm{B}$ " in Figure $\mathrm{O}$, has cables laced and overlapped only on the bottom of the baskets. The change between Wall A and B came with the hope of minimizing flexibility in the structure by limiting cable deformation. In having the cables overlap on the bottom of the basket, the material that fills the basket will have essentially 'pinned' the cables in place and limited movement in dynamic response.

The third wall, labeled "C" in Figure O above, was designed to minimize the need for hand ties by lacing the cables almost entirely on the exterior of the baskets. Also, it was intended to better utilize the basket wire by maximizing tension on the vertical 
components of the basket, rather than the horizontal. By running the cables through the apertures in the basket mesh, the cables are not only held in place at the 90-degree bends required, but can also be externally tightened after installation. The only ties required are those at the very top of the basket to keep the cables in tension since there is nothing resisting cable movement as in the first two wall designs.

\subsection{Forced Vibration Test Equipment}

This section reviews the materials required to perform the experiment. Three specific types of equipment are required to run a dynamic analysis on the walls: a mass shaker, accelerometers, and standard lab software, Math Works Inc. 2009. Initially, the shake table was also to be part of the testing, but further research into the table's behavior made that impossible, as explained below.

\subsubsection{Shake Table}

The shake table would have provided significant insight into the behavior of gabion walls in regard to ground motion responses. However, when the hydraulic pump shaft is disconnected from the table and tested on

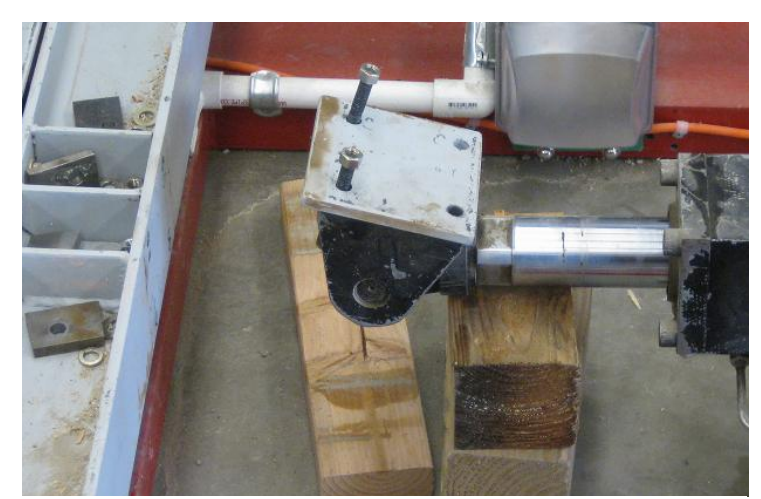

Figure P - Disconnected hydraulic pump its own (see Figure P, right) to check if an accurate performance was even possible, the pump failed to put out a purely sinusoidal signal. This failure made results from the table's performance unusable. 


\subsubsection{Mass Shaker}

The $100 \mathrm{lbs}$. shaker is portable and can force a reasonably constant sinusoidal force of $30 \mathrm{lbs}$. The available frequency range is between $2-20 \mathrm{~Hz}$. Because the forces caused by the shaker are relatively small, the friction between the shaker and the involved structure is sufficient to make additional mechanical anchorage unnecessary (McDaniel and Archer, 2009).

\subsubsection{Accelerometers}

Piezoelectric flexural accelerometers were used to measure the motions of the structure. They can capture frequency results from $1-200 \mathrm{~Hz}$ and beyond, while their range for measurement can be upwards of 0.5g. A standard 16-bit analog converter was insufficient for previous testing, so a 24-bit device able to handle up to 4 accelerometers was employed as its replacement (McDaniel and Archer, 2009).

\subsubsection{Lab Software}

The results from the accelerometers are processed using lab software called Math Works Inc. (2009). The software captures the desired frequencies, while filtering out the inapplicable lower and higher frequencies. The software then completes a Fast-Fourier Transform (FFT) of the data, and displays it alongside the raw, captured data. This time history view is required for deciphering between relative signs of the displacements of the structure, either positive or negative. The peaks from the FFT plot are pulled and shown on-screen to assist in result processing. The equipment can be seen in Figure Q on the following page (McDaniel and Archer, 2009). 


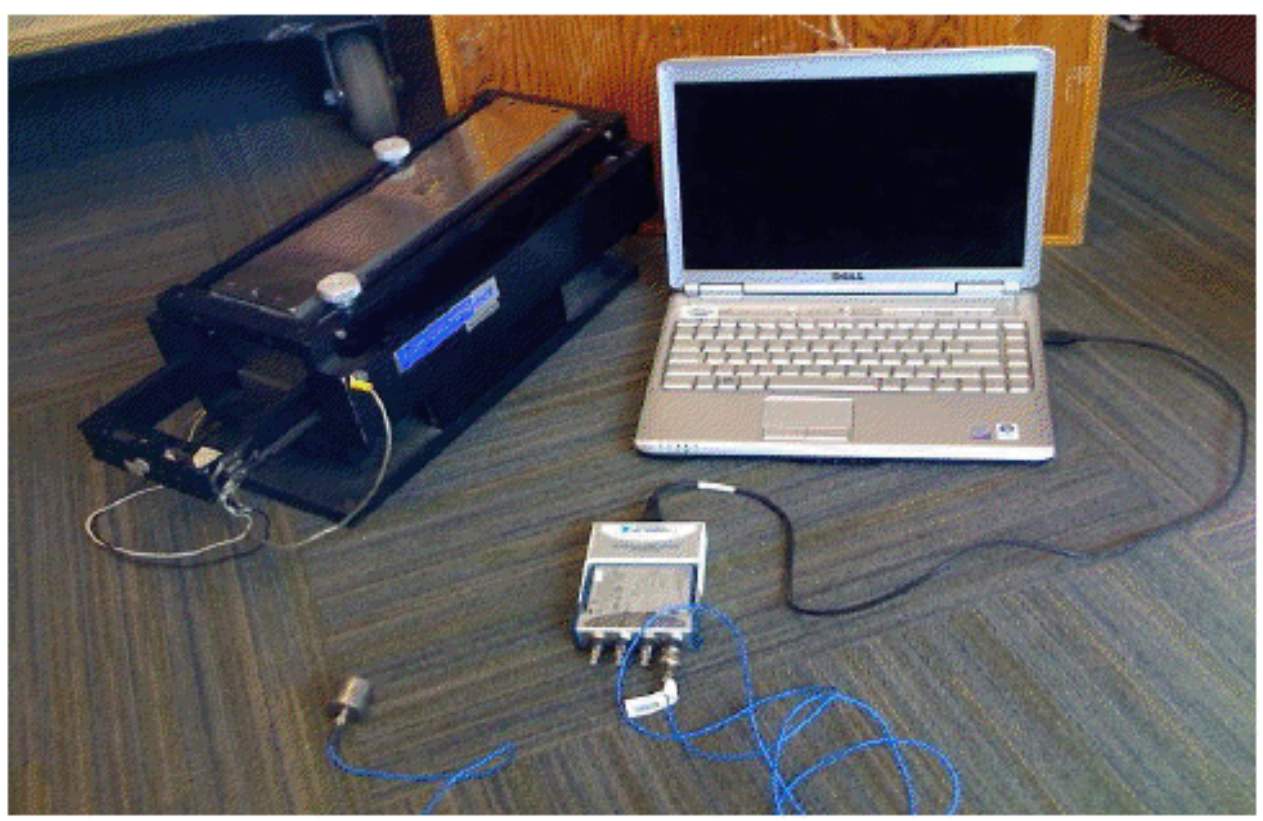

Figure Q - Testing equipment (McDaniel and Archer, 2009). 


\subsection{VIBRATION STUDY}

The following section will deal mainly with the experimental data: data gathering, processing and interpretation in regard to dynamic behavior.

\subsection{Experimental Procedure and Data Reduction}

Though the cable patterns for the walls differ, it was very important to keep the testing of each wall precise and standardized. In order to do this, a list of tests is created to keep the demand on each wall and the results consistent. The following tests were conducted, with each test to be expanded on in its respective section below: frequency sweeps, tone extractions, mode shape analysis at the natural frequencies, and snap-back tests (where applicable).

\subsubsection{Frequency Sweeps}

To capture a wall's behavior at various frequencies, a type of testing called a frequency sweep is required. To conduct a frequency sweep, the mass shaker is programmed to begin at a small frequency and gradually increase the magnitude of frequency it is forcing until reaching the final, higher programmed frequency. This range is initially in the magnitude of $2 \mathrm{~Hz}-22 \mathrm{~Hz}$ in the effort to capture a large range of behaviors, but testing at this large of an interval produces inconsistent results. The solution to this problem is to set the shaker to sweep at approximately $10 \mathrm{~Hz}$ intervals at a time, with mild overlap between tests. This interval will be on the order of a $2 \mathrm{~Hz}-12$ $\mathrm{Hz}$ sweep, while the next range might be from $10 \mathrm{~Hz}-19 \mathrm{~Hz}$, until the entire range of behaviors is captured. 


\subsubsection{Ambient Vibration}

Before any dynamic testing begins on the wall, two accelerometers are set up: one on the shake table, and one on the top of the structure. The accelerometer on the shake table is assumed to capture any ambient frequencies as experienced by the environment, while the accelerometer on the wall captures the ambient vibrations as experienced by the wall. An example of ambient vibration can be seen below in Figure Q. It should be noted that though there are three notable spikes in this graph, the one occurring at approximately $12 \mathrm{~Hz}$ is not attributed to the wall system itself, but possibly the natural frequency of the shake table itself. This assumption was confirmed when the spike almost disappeared once the structure was set in motion.

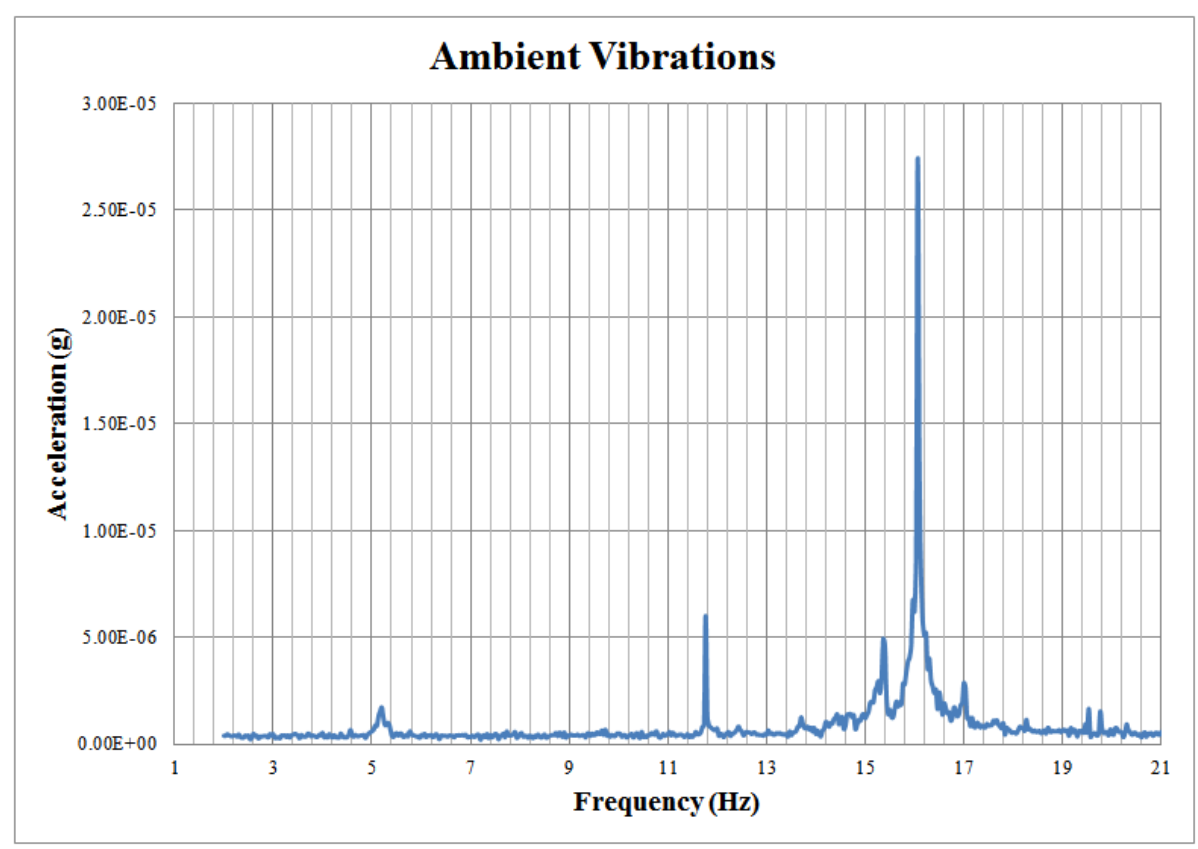

Figure R - Lab software output from Wall A Testing 


\subsubsection{Wall Frequency Sweeps}

After collecting the ambient data, the mass shaker is programmed at a "start" and "stop" frequency for the desired sweep range over a set time of 30 seconds. The lab software is then activated to record and transform the accelerometer's data. One important detail of the software was that it is allowed to record the data from the accelerometers but has a "filter" in the system that averages the cycle of data it receives. For example, assume the shaker is set to sweep increasingly from 2 to $10 \mathrm{~Hz}$ over a period of 30 seconds. The wall behavior during those 30 seconds will be transmitted by the accelerometers to the computer, which will take that data and average it with the next 30 seconds of data it receives, etcetera. This averaging process can occur up to 10 times and is useful for removing non-useful data during periods when a loud noise or movement causes an artificial spike in the graph.

An interesting behavior that occurs involves the interaction between the shaker and the table. Though the table is thousands of pounds heavier than the shaker, the table still has a natural frequency of its own. This frequency is even more noticeable since the hydraulic pump has been disconnected from the table, enabling more free motion than before. Some of the more ambiguous spikes in the graph not attributed to the structure may be attributed to this phenomenon (for example, those around $12 \mathrm{~Hz}$ ). This cannot be said definitely since the table was not measured independent from a structure.

After the observed sweep results are consistent from averaging cycle to averaging cycle, then are considered final and exported into Excel. Once in Excel, the sweeps are 
superimposed on each other to create a full-range frequency report. From this created graph, obvious spikes are observable, as in the ambient vibrations graph. These spikes represent natural frequencies of the structure, and once identified, can be investigated on a much finer level. An example of a superimposed frequency sweep of Wall A can be found below in Figure R.

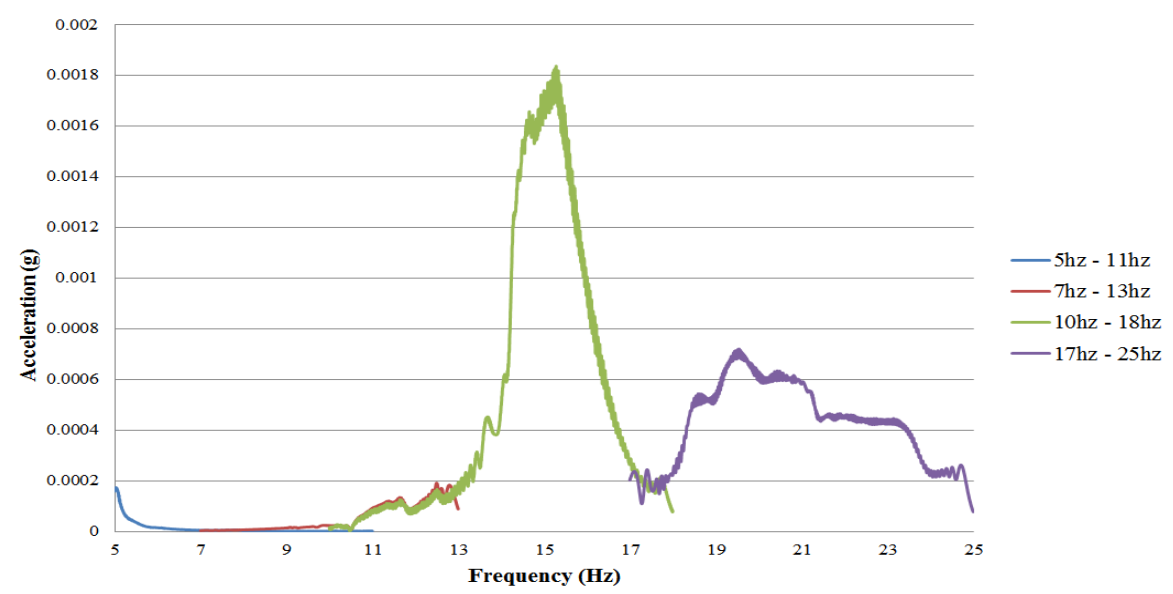

Figure S - Superimposed frequency sweep for Wall A

An important thing to notice is what occurs in the overlap of two sweep segments.

The joining of two segments should be virtually flawless, with one segment picking up very near where the last segment left off. This can be observed here in Figure R, especially in the frequency range of $11 \mathrm{~Hz}-13 \mathrm{~Hz}$.

There are two consistent peaks in each wall's data, one around $5 \mathrm{~Hz}$ and one around $15 \mathrm{~Hz}$ (as seen in Figure $\mathrm{R}$ on the previous page). Though activity is observed from $19 \mathrm{~Hz}-25 \mathrm{~Hz}$, the magnitude is not as defined as that seen around $15 \mathrm{~Hz}$ and is therefore eliminated as a potential range requiring further investigation. 


\subsubsection{Modal Frequencies (Tone Extraction)}

In order to further investigate the areas of peaked activity found in the full-range frequency sweep, smaller modal sweeps are done. A modal sweep, or "tone extraction", involves programming the shaker to force a single-frequency vibration, while recording the structure's response. The frequency is then changed at a very small interval, anywhere from $0.1 \mathrm{~Hz}$ to $0.25 \mathrm{~Hz}$, to record behaviors on both sides of the activity peaks. After the frequencies are recorded and plotted, a best-fit curve is used to connect the dots as smoothly as possible. The result of tone extraction for the first mode of Wall $\mathrm{C}$ can be seen below in Figure T. Another name for the frequency peak is the "natural" or "resonant" frequency. Chopra defines this frequency as "the forcing frequency at which the largest response amplitude occurs" (Chopra, 2007).

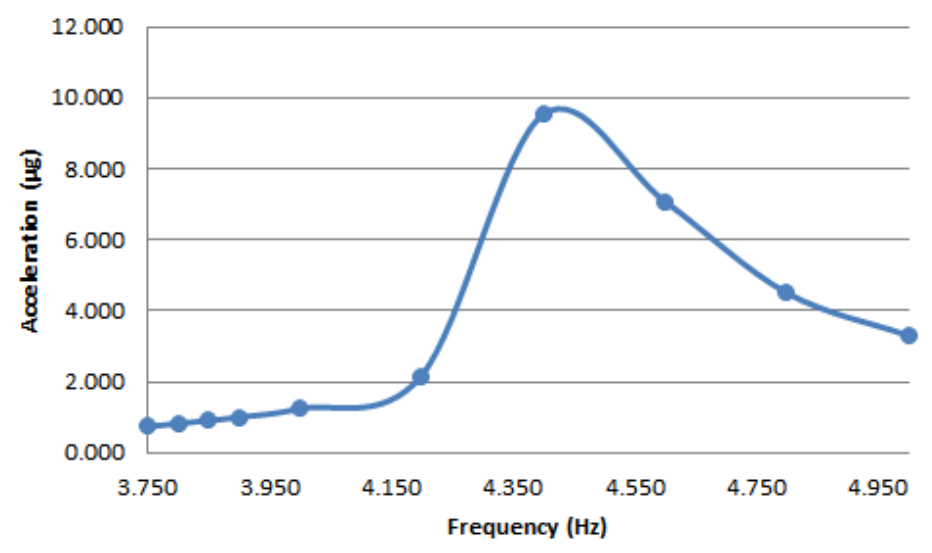

Figure T - Tone Extraction for Wall C

As seen in Figure $\mathrm{T}$ above, points are taken to the left and right of the measured resonant frequency to re-create the peak first seen on the large-scale sweep. This test allows a closer look into the structure's behavior, and results in at least two vital pieces of 
information needed for further investigation: the exact frequency the peak occurred at, and the slopes towards and away from the resonant peak. The importance of both of these pieces of information will be discussed in the following two sub-sections.

\subsubsection{Damping Ratios}

Every structure has a "damping ratio", that structure's measured dissipation of energy. Damping itself is amplitude-dependent, so this property is heavily investigated in the specific area of seismicity. For this set of experiments, two methods were chosen to investigate the individual damping ratios of the walls: a "half-power bandwidth" and a "pull-back test."

A half-power bandwidth test requires the graph created from the tone extraction process, also known as a "frequency response curve." The peak of the frequency curve is divided by $\sqrt{2}$, and a horizontal line is drawn across the graph at that value. This line intersects the curve at either side of the peak, and those two intersections are labeled $f_{a}$ and $f_{b}$, respectively. The area between these two intersections is known as the half-power bandwidth (Chopra, 2007). Figure $\mathrm{U}$ on the following page shows this process of finding the half-power bandwidth for Wall C's first mode.

FREE-STANDING GABIONS IN SEISMIC REGIONS 


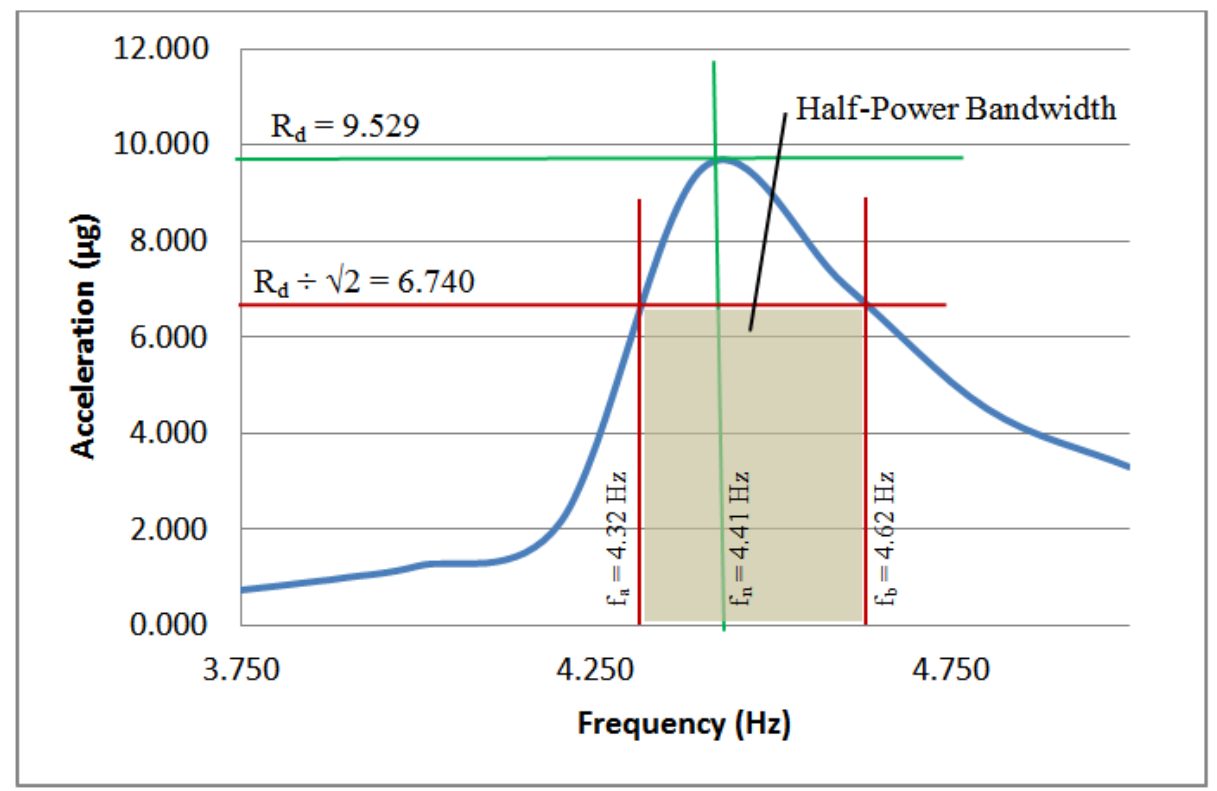

Figure U - Half-Power Bandwidth Process

Through a system of derivations that will not be recounted here, the final equation for retrieving the damping ratio $(\xi)$ is seen below.

$$
\xi=\frac{f_{b}-f_{a}}{2 f_{n}}
$$

where $\quad f_{b}$ is the greater half-power frequency $(\mathrm{Hz})$, $f_{a}$ is the lesser half-power frequency $(\mathrm{Hz})$, and $f_{n}$ is the natural, or resonant, frequency $(\mathrm{Hz})$.

Using the respective values of $f_{a}, f_{b}$ and $f_{n}$ for Wall $\mathrm{C}$, from Figure $\mathrm{U}$ on the previous page, the damping ratio is $3.63 \%$. However, the correct type of frequency curve has a sharp slope leading up the peak on both sides, like the left side of Wall C's curve. For a more correct damping calculation, "half half-power bandwidth" was proposed, where the side that displays the correct form is mirrored over and the damping calculation 
re-done. This idea is displayed below in Figure V. The exact cause of the nonsymmetrical tone extraction is unknown; however, it may be that there is a residual energy in the structure once the structure resonates at a fundamental frequency and does not taper off immediately after excitation.

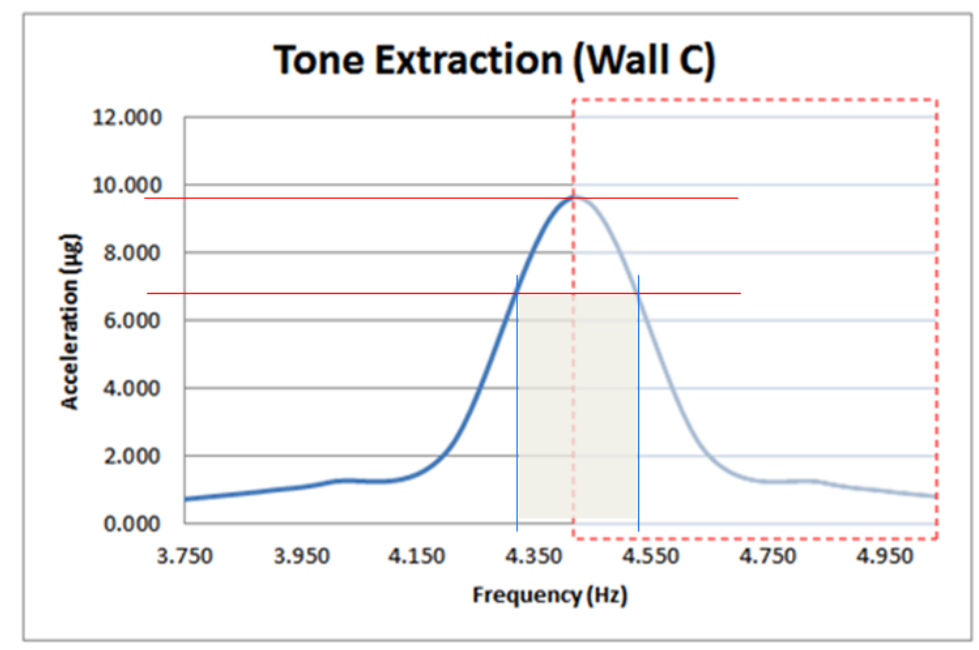

Figure V - "Half Half-Power Bandwidth" Process

Using the mirrored image for the calculations, the damping ratio decreases to 2.04\%. Taking the damping ratio from the complete half-power bandwidth with that of the half half-power bandwidth, the damping ratio was taken as $2.85 \%$ averaged. Wall A had the same issue with form as Wall C did: both walls' slopes were not smooth or steep enough to qualify for the half-power bandwidth method. However, Wall A had already been tested and deconstructed by the time of this realization, so the results for both the half-power and the half half-power bandwidth were averaged for a damping ratio. Using 
Equation 1 from page 27, the damping ratio for the half-power is $5.08 \%$, while the half half-power yields a $3.93 \%$ damping ratio. These two values average to $4.5 \%$ for Wall A.

Wall B's frequency curves lacked the form required for the half-power bandwidth procedure also. Because of this lack, Wall B required another test to give primary values for a damping ratio: a snap-back test.

A snap-back test, as the name would suggest, involves pulling back on the structure and measuring its "snapped" behavior once the restraints are removed suddenly. Wall B and Wall C were tested this way when the half-power bandwidth test could not accurately measure the damping ratios. The process involves lacing wire through the top basket of the wall, attaching it to a digital force scale, and pulling back the structure until the scale read $40 \mathrm{lbs}$. Once at $40 \mathrm{lbs}$. of force, the lab software begins recording from an accelerometer placed on the very top of the structure, and the wire pulling the structure is cut. The wall is then allowed to oscillate naturally, and the results are exported for further analysis. For these specific results, the raw data is required versus having the software complete a Fast-Fourier Transform (FFT). An example of the raw snap-back data from Wall B can be seen on the next page in Figure W.

The shake table has the potential to absorb energy from the snap-back test, but the interference was assumed to minimal and thus, dismissed as a concern. 


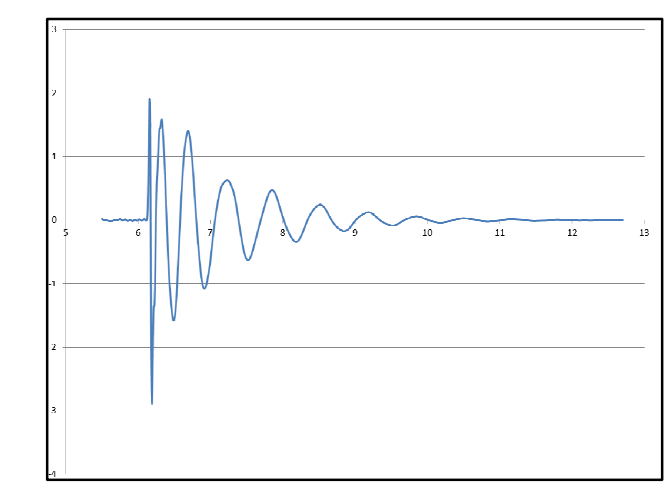

Figure W - Oscillatory results from Wall B

The damping ratio was collected from these results as well, by measuring the decay from one peak to another. The equation for this calculation can be seen below (Chopra, 2007).

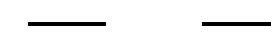

Eq. 2

where $\quad u_{i}$ is the amplitude of the first chosen extremity for analysis, $j$ is how many peaks away the second chosen extremity is, and $u_{i+j}$ is the amplitude of the second chosen extremity for analysis.

This procedure can be done for the initial, large-amplitude oscillations and also the smaller oscillations that occur after the structure's motion has damped out. This variance in application can be useful in comparing accuracy in data since both results should be relatively close to each other. Examples of how the variables from Equation 2 are applied can be seen in Figure $\mathrm{X}$ on the following page. 


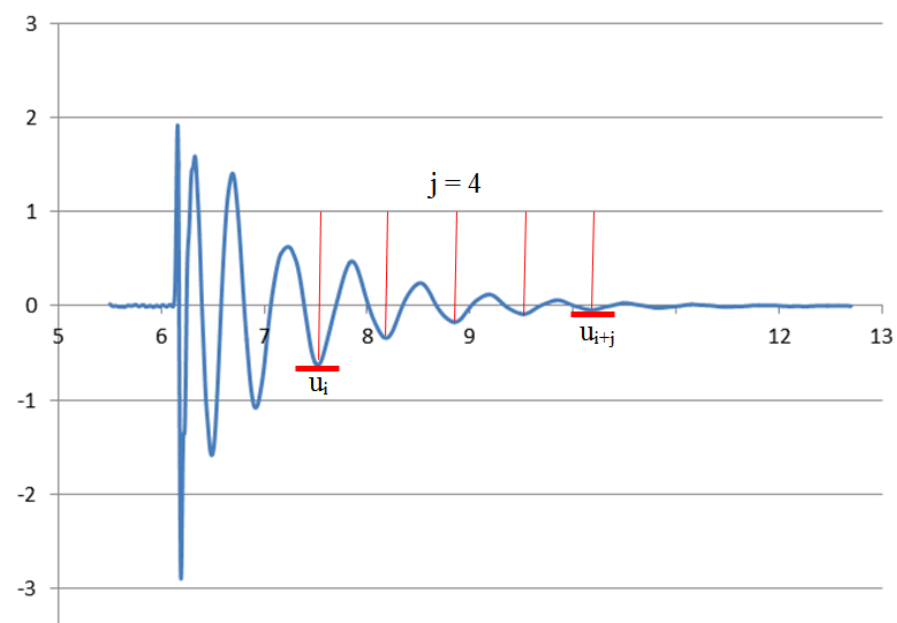

Figure X - Damping through oscillatory decay measurement

Equation 2 is applied to the results from Wall B and Wall C, both to the amplitude directly after snapping back, and then also to the amplitude after the wall had oscillated for a few seconds. For Wall B, large-amplitude data yields a damping ratio of 11.12\%, while the small-amplitude data yields a $10.77 \%$ damping ratio. These two values averaged give Wall B a damping ratio of approximately $11 \%$. Wall C's large-amplitude damping ratio is $8.65 \%$, while the small-amplitude data yields a $9.82 \%$ damping ratio. The average of these two values gives Wall $\mathrm{C}$ an approximate damping ratio of 9.3\%.

These higher damping values are due to the method of testing: the shaker causes small displacements, while the snap-back test causes large displacements. Since the over-all nature of this experiment is to test for earthquake compatibility, the larger damping ratios are more appropriate for analysis. Wall A's damping ratio of $4.5 \%$ from the half- and half half-power bandwidth procedure is not an accurate portrayal of largedisplacement damping. Therefore, a damping ratio of $9.3 \%$ from Wall C's results will be 
taken instead for a more accurate analysis of performance. I chose this method because both Wall B and C experienced relatively similar damping ratios despite their design differences, meaning Wall A would most likely experience a large-displacement damping ratio of this magnitude as well.

\subsubsection{Mode Shapes}

As mentioned in Modal Frequencies (Section 4.1.2), the other piece of information gathered from a tone extraction is the exact frequency at which a mode occurs. Modal frequencies are unique to each structure and rely heavily on the way the structures were constructed. If the structure is excited at its exact modal frequency, a significantly higher level of activity can be observed within the structure. Also, the modal frequency can determine what sort of an earthquake the structure will respond to, thus aiding in determining what types of structures should be built in areas with a known seismicity pattern.

There is an indefinite number of modes for every structure, though most occur at so high a frequency that they are never investigated. The first mode occurs at the lowest frequency and experiences only single-bend behavior. The second mode occurs at the next highest modal frequency, and experiences double curvature. For more clarity in shapes, the un-swept mode shapes from Wall A can be seen below in Figure X. 

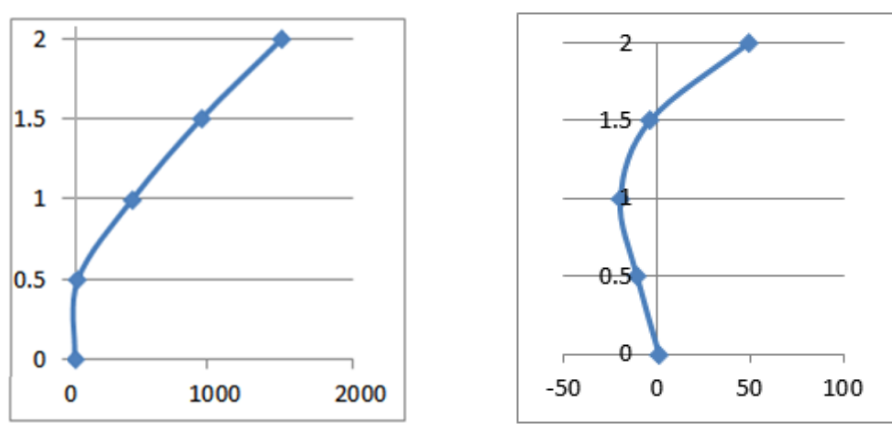

Figure Y-Apparent First and Second Mode Shapes (left, right respectively)

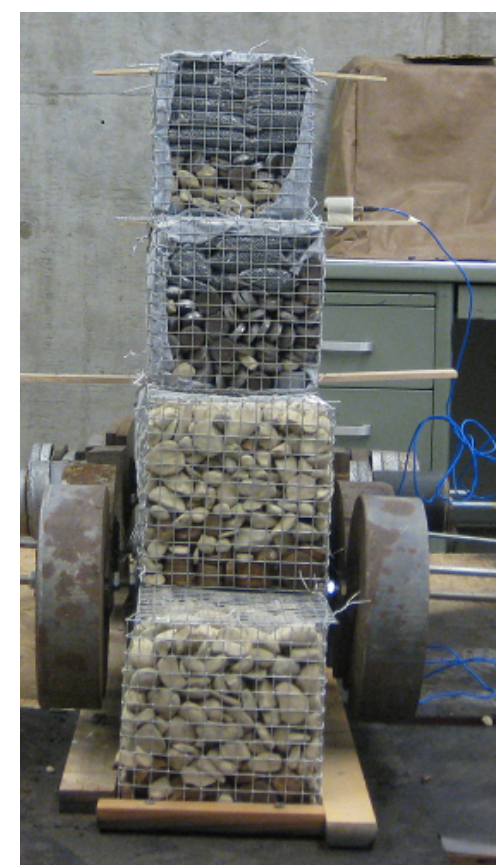

Figure Z - Constructed

Wall Elevation

It should be noted that the two mode shapes in Figure $\mathrm{X}$ on the previous page are raw, un-swept mode shapes, meaning that the values shown here are not solely influenced by the first or second mode, respectively, but rather are influenced by all the modes the structure experiences. This idea of "sweeping" will be further discussed in Modal Sweeping (Section 6.3).

For these experiments, the first two modes are identified from the tone extractions, and are found to occur at $5 \mathrm{~Hz}$ and $15 \mathrm{~Hz}$, approximately. For each of the walls, the shaker is set to consistently put out either of the two frequencies, and the wall's behavior is recorded using accelerometers. There are two accelerometers used in the testing set-up: one on the shake table itself, and one on the structure. When investigating mode shapes, having an accelerometer on the table allows confirmation that the shaker is indeed putting out the prescribed frequency. 
The other accelerometer, then, is free to investigate the structure's behavior, accomplished by moving the accelerometer from one paddle to another along the height of the wall (see Figure Y above). At each frequency, the accelerometer gathers no fewer than 4 sets of data from each level along the wall height to ensure consistent results. These results are then averaged for the final values of the mode shapes.

The data used for the modal investigation are raw (versus the data that went through the FFT). This "raw" state is important because the phase of the mode can also be determined. If the accelerometers on both the table and the wall are in-phase, then the waves displayed on the lab software are synchronized in regard to their peaks and valleys. However, if the peaks and valleys are out of phase, this shows that the table and the wall are moving in opposite directions and thus, a negative is assigned to whatever value is recorded at that level. An example of the out-of-phase case can be seen on the following page in Figure AA. 


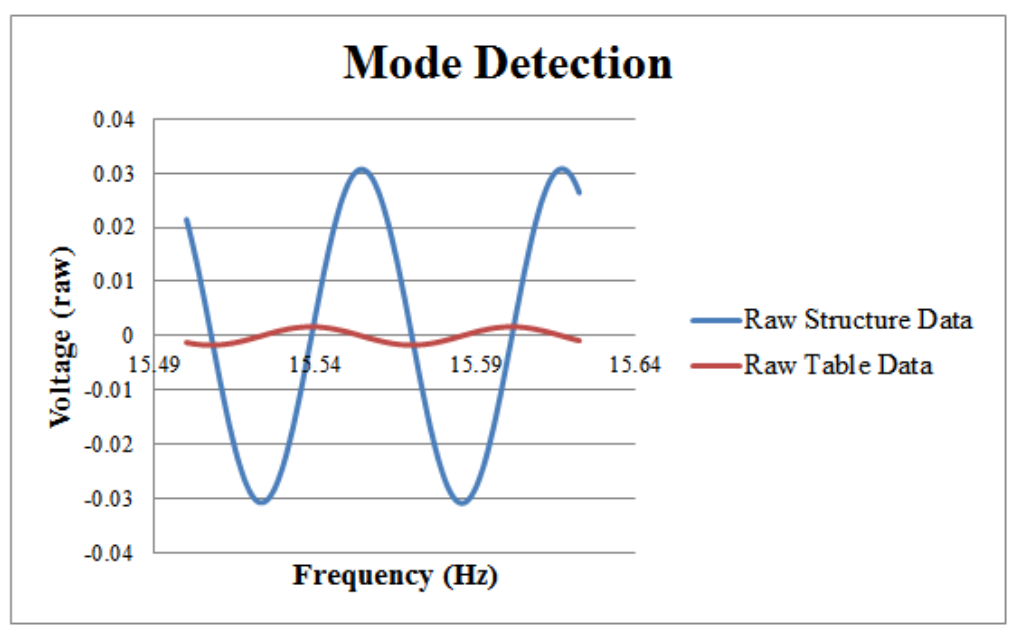

Figure AA - Lab results for synchronizing check

This observation of phase difference also allows for determining whether the frequencies assumed truly are the resonant frequencies. If they are correct, then the results should mimic the results seen in Figure $X$ from page 31 . The first mode should have only single curvature, whereas the second mode should experience only double curvature.

\subsubsection{Discussion of Results}

As mentioned above, the first two resonant frequencies for each wall are gathered from the frequency sweeps and tone extraction. The structures are then observed at each of these frequencies along their entire height to measure their modal performance. The results for each of the walls can be found below. Note that all mode shapes are in units of milli-g's. 
$\underline{\text { Wall A }}$

$$
\begin{array}{rc}
\omega_{\mathrm{n}-1}=4.90 \mathrm{~Hz} & \omega_{\mathrm{n}-2}=15.25 \mathrm{~Hz} \\
\phi_{1}=\left[\begin{array}{l}
1.581 \\
0.975 \\
0.502 \\
0.120
\end{array}\right] & \phi_{2}=\left[\begin{array}{c}
49.288 \\
-4.060 \\
-19.640 \\
-10.735
\end{array}\right]
\end{array}
$$

$\underline{\text { Wall B }}$

$$
\begin{array}{cc}
\omega_{\mathrm{n}-1}=4.60 \mathrm{~Hz} & \omega_{\mathrm{n}-2}=15.20 \mathrm{~Hz} \\
\phi_{1}=\left[\begin{array}{c}
23.293 \\
14.905 \\
7.519 \\
3.039
\end{array}\right] & \phi_{2}=\left[\begin{array}{c}
80.180 \\
-4.769 \\
-38.603 \\
-17.587
\end{array}\right]
\end{array}
$$

$\underline{\text { Wall C }}$

$$
\begin{array}{cc}
\omega_{\mathrm{n}-1}=4.40 \mathrm{~Hz} & \omega_{\mathrm{n}-2}=14.30 \mathrm{~Hz} \\
\phi_{1}=\left[\begin{array}{l}
6.728 \\
6.234 \\
3.169 \\
1.058
\end{array}\right] & \phi_{2}=\left[\begin{array}{c}
51.836 \\
-6.150 \\
-20.515 \\
-14.882
\end{array}\right]
\end{array}
$$




\subsection{CREATION OF DESIGN SPECTRA}

This section of the thesis deals with the geographical application of the gabion wall system, in other words, investigating the behavior of the area this system would be applied to regarding seismicity. Ideally, this system can apply to construction in a developing nation that currently relies on construction options like adobe brick. This thesis looks at Kenya due to the available seismic information surrounding the country. The next step is to investigate known seismic activity in the area and create a series of design spectra that will help predict the individual wall's behavior.

\subsection{Explanation of Design Spectra}

A design spectrum is frequently represented as a "tri-partite" graph featuring the natural vibration period, $\mathrm{T}_{\mathrm{n}}$, against 3 logarithmic axes of Deformation, Pseudo-Velocity, and Pseudo-Acceleration. The actual plot on the graph is a set of data representing characteristic behavior of the geographical region. Similarly, the constant portions of the plot for the Displacement, Pseudo-Velocity, and Pseudo-Acceleration axes are dependent solely on the seismic behavior of the area. The axis measuring the natural vibration period, then, allows this information to become applicable to the structural system under investigation. A blank tripartite graph can be seen on the following page. Note that this particular graph has the natural vibration period as its x-axis. Frequency and periods are

inversely proportional; that is, $T_{n}=\frac{1}{f_{n}}$, so the frequency values gathered during experimentation can easily be converted to natural vibrating periods. 


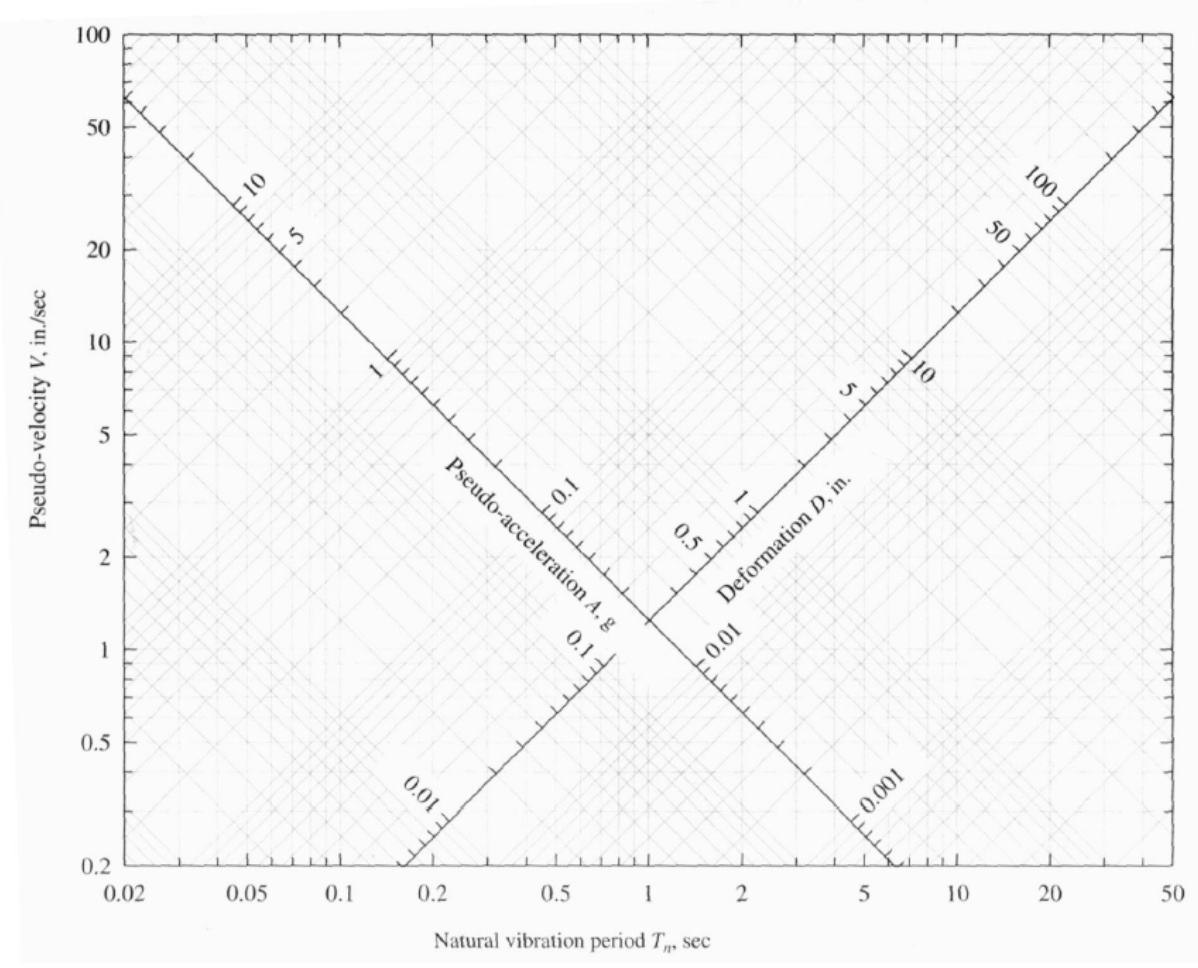

Figure BB - Tripartite Graph (Chopra, 2007)

By locating the structure's period on the x-axis, the expected deformation, pseudo-velocity, and pseudo-acceleration can be gathered for further analysis. This process will be described in further detail in the sections to follow.

\subsection{Processing Earthquake Records}

One of the first steps in creating a design spectrum is to gather data from the three worst earthquakes in the area, or average the information from seven different earthquakes and assume this result is the typical behavior for the area. Kenya is located on the eastern coast of Africa, and does have recorded seismic activity. However, data for a set of seven earthquakes specifically in Kenya cannot be located through the United 
States Geological Survey (USGS) since its research pertains mainly to the United States.

The compromise for this discrepancy is to take the earthquake data available for the countries in closest proximity. As seen in Figure CC below, Kenya shares a border with Ethiopia, Uganda, and Tanzania. The USGS has earthquake data for each of these countries, so a suite of seven earthquakes is available for analysis (USGS, online).

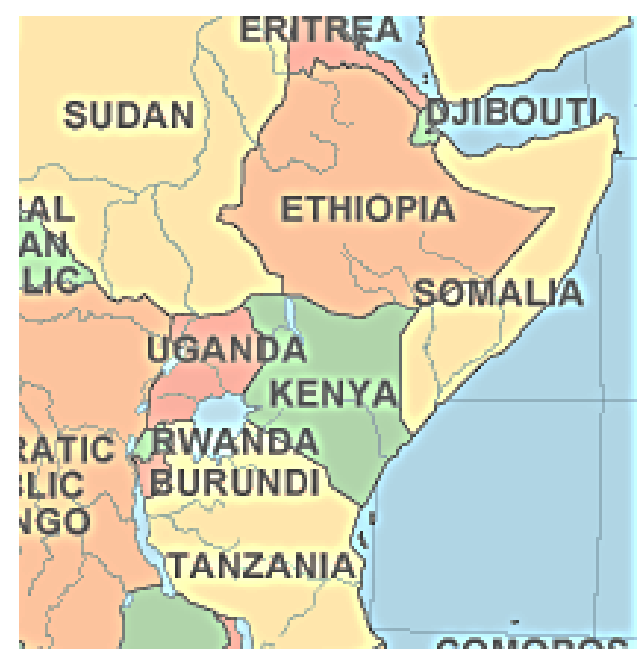

Figure CC - Excerpt from map of Africa (http://www.world-atlas.us/africa.htm)

Each set of earthquake data located through the USGS has been processed and summarized. The four most pertinent pieces of information for this analysis are the dates of the individual earthquakes, their locations, the peak ground accelerations (PGA), and the peak ground velocities (PGV). Table 1 on the following page summarizes these pieces of information for each of the earthquakes. 
Table 1 - Earthquake Data Summary (Source: USGS)

\begin{tabular}{|c|c|c|c|c|}
\cline { 2 - 5 } \multicolumn{1}{c|}{} & Country & Date & PGA (\%g) & PGV $(\mathbf{c m} / \mathbf{s})$ \\
\hline 1 & Uganda & $10 / 9 / 1991$ & 0.29053 & 6.4432 \\
\hline 2 & Uganda & $2 / 5 / 1994$ & 0.34642 & 7.8731 \\
\hline 3 & Tanzania & $8 / 3 / 2010$ & 0.19246 & 3.2905 \\
\hline 4 & Tanzania & $10 / 2 / 2000$ & 0.27283 & 13.874 \\
\hline 5 & Tanzania & $8 / 18 / 1994$ & 0.25239 & 14.304 \\
\hline 6 & Ethiopia & $8 / 21 / 1989$ & 0.33222 & 7.694 \\
\hline 7 & Ethiopia & $9 / 21 / 1993$ & 0.25662 & 7.1231 \\
\cline { 2 - 4 } & \multicolumn{4}{|c|}{ Average: } \\
\cline { 2 - 4 }
\end{tabular}

As seen in the collected data in Table 1 above, the average PGV is $8.66 \mathrm{~cm} / \mathrm{sec}$ (3.160 in/sec), which is a very low number for creation of a design spectrum. Newmark and Hall put forward an alternate method for gathering the PGV values, especially in cases when actual PGV may not be available (Newmark and Hall, 1982). The PGV values can be created by multiplying the PGA values by $48 \mathrm{in} / \mathrm{sec}$. Applying this method to the PGA values gathered from the USGS data, the average PGV value then becomes $13.327 \mathrm{in} / \mathrm{sec}$, a much better value for spectrum creation. This application will become more obvious in the development procedures to follow in this section.

\subsection{Determination of Spectrum Coefficients}

The period axis is not the only way the design spectrum is specialized for a structure-Newmark and Hall also propose a method for modifying the constant values on the Deformation, Pseudo-Velocity, and Pseudo-Acceleration axes (velocity and acceleration values are termed "Pseudo-" because they are derived from and dependent 
on the Deformation values). In their method, each of the values for the three separate regions is multiplied by a coefficient that is dependent on the structure's damping ratio as discussed in Damping Ratios (Section 4.1.3). The table containing these coefficients, referred to as "Spectrum Amplification Factors for Horizontal Elastic Response" in Newmark and Hall's book, has been re-created below in Table 2 for convenience.

Table 2 - Spectrum Amplification Factors (Newmark and Hall, 1982)

\begin{tabular}{cccc}
\hline Damping, & \multicolumn{3}{c}{ Median (50\%) } \\
\cline { 2 - 4 } \% Critical & $\mathrm{A}$ & $\mathrm{V}$ & $\mathrm{D}$ \\
\hline 0.5 & 3.68 & 2.59 & 2.01 \\
1 & 3.21 & 2.31 & 1.82 \\
2 & 2.74 & 2.03 & 1.63 \\
3 & 2.46 & 1.86 & 1.52 \\
5 & 2.12 & 1.65 & 1.39 \\
7 & 1.89 & 1.51 & 1.29 \\
10 & 1.64 & 1.37 & 1.20 \\
20 & 1.17 & 1.08 & 1.01 \\
\hline
\end{tabular}

Obviously, the critical damping for each structure does not fall directly on the given values given in Table 2 above, so the exact value for each of the coefficients must be attained by linear interpolation. The symbol typically given to these coefficients is $\alpha$, with the specific region noted in the subscript. For example, a coefficient describing the spectrum amplification factor for the pseudo-acceleration region will be denoted as $\alpha_{A}$. Table 3 on the following page summarizes the critical damping values for each of the walls, in addition to the coefficients retrieved by linearly interpolating the values from Table 2 above. 
Table 3 - Amplification Factor Summary

\begin{tabular}{|c|c|c|c|}
\cline { 2 - 4 } \multicolumn{1}{c|}{} & $\begin{array}{c}\text { Damping, } \\
\text { \% Critical }\end{array}$ & $\boldsymbol{\alpha}_{\mathrm{A}}$ & $\boldsymbol{\alpha}_{\mathrm{V}}$ \\
\hline Wall A & 9.30 & 1.580 & 1.343 \\
\hline Wall B & 11.00 & 1.217 & 1.109 \\
\hline Wall C & 9.30 & 1.580 & 1.343 \\
\hline
\end{tabular}

As discussed in the previous section, the average maximum PGA, $A_{g}$, is $0.2776 \mathrm{~g}$, and the PGV, $V_{g}$, per Newmark \& Hall's method is $13.327 \mathrm{in} / \mathrm{sec}$. Each of these two values can be multiplied by the appropriate Spectrum Coefficient in Table 3 above to find the modified spectrum values. The result of this calculation can be found in Table 4 below.

Table 4 - Amplified Spectrum Results

\begin{tabular}{|c|c|c|}
\cline { 2 - 3 } \multicolumn{1}{c|}{} & $\max _{\mathbf{~}}$ & \\
\hline Wall A & $0.439 \mathrm{~g}$ & $17.898 \mathrm{in} / \mathrm{sec}$ \\
\hline Wall B & $0.338 \mathrm{~g}$ & $14.780 \mathrm{in} / \mathrm{sec}$ \\
\hline Wall C & $0.439 \mathrm{~g}$ & $17.898 \mathrm{in} / \mathrm{sec}$ \\
\hline
\end{tabular}

These values can then all be plotted on a tri-partite graph to create a design spectrum.

\subsection{Creation of Mode-Specific Design Spectra}

As stated above, the values from the earthquake records (both raw and modified with Newmark \& Hall's coefficients), all come together to create the final design 
spectrum, which can then be used to predict behavior of a structure based on the previously-measured periods. Table 5 below summarizes the values for frequencies, calculated periods, and spectrum values for each of the three walls.

Table 5 - Summary of Wall Data for Spectrum Creation

\begin{tabular}{|c|c|c|c|c|c|}
\hline & & Frequency $(\mathrm{Hz})$ & Period (sec) & $\max A_{g} * \alpha_{A}$ & $\max A_{g} * \alpha_{V}$ \\
\hline \multirow{2}{*}{ Wall A } & Mode 1 & 5.02 & 0.199 & \multirow{2}{*}{$0.439 \mathrm{~g}$} & \multirow{2}{*}{$17.898 \mathrm{in} / \mathrm{sec}$} \\
\hline & Mode 2 & 15.25 & 0.066 & & \\
\hline \multirow{2}{*}{ Wall B } & Mode 1 & 4.60 & 0.217 & \multirow{2}{*}{$0.338 \mathrm{~g}$} & \multirow{2}{*}{$14.780 \mathrm{in} / \mathrm{sec}$} \\
\hline & Mode 2 & 15.20 & 0.066 & & \\
\hline \multirow{2}{*}{ Wall C } & Mode 1 & 4.40 & 0.277 & \multirow{2}{*}{$0.439 \mathrm{~g}$} & \multirow{2}{*}{$17.898 \mathrm{in} / \mathrm{sec}$} \\
\hline & Mode 2 & 14.40 & 0.069 & & \\
\hline
\end{tabular}

Because each of the walls has unique damping ratios, the walls each require their own design spectrum for analysis. The values from Wall A will be used to demonstrate how to create the design spectrum.

\subsubsection{Creating the Constant Spectrum Regions}

For all walls, $\mathrm{A}_{\mathrm{g}}=0.2276$ and $\mathrm{V}_{\mathrm{g}}=13.327 \mathrm{in} / \mathrm{sec}$. These two constant values are shown in Figure DD on the following page with red and blue lines, respectively. Note that each of the lines terminates at the intersection of two lines for a solid "corner" of the graph, denoting a transition between acceleration- or velocity-dominated behaviors. 


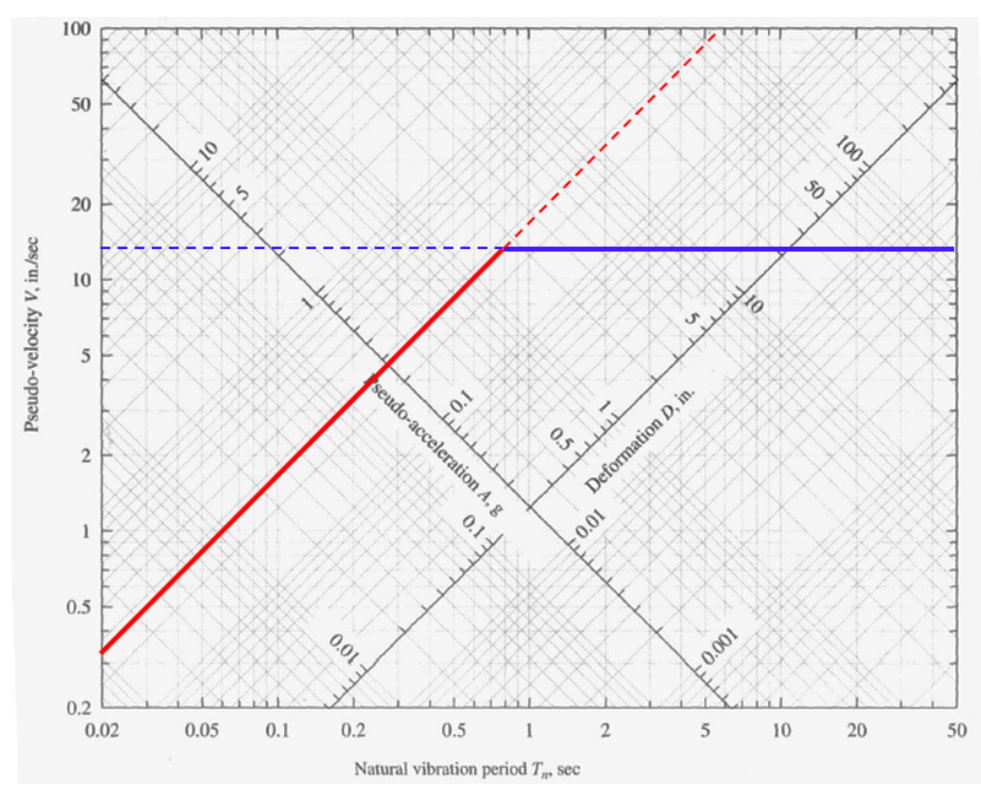

Figure DD - Beginning stages of design spectrum creation

Recall that the frequencies for Wall A are $5.02 \mathrm{~Hz}$ and $15.25 \mathrm{~Hz}$. Looking at the current graph, both of these values fall in the areas already developed in the acceleration and velocity regions of the spectrum; therefore, there is no need to develop the deformation portion of the spectrum.

\subsubsection{Creating the Amplified Spectrum Regions}

The next step is to plot the design spectrum again with the amplification factors included. These two plots will be drawn on the same graph for the sake of comparison. The initial points of transition from one type of spectrum value to another (for example, acceleration to velocity regions) are set despite structure specifics. For periods up to $T=$ $0.035 \mathrm{sec}$, the unmodified spectrum value is used. For a period between $0.035 \mathrm{sec}$ and $0.125 \mathrm{sec}$, the spectrum transitions from unmodified to modified values. After $T=0.125$ 
sec, the intersection of the two amplified regions govern the final "corner" in the spectrum. See Figure EE below for a graphical interpretation.

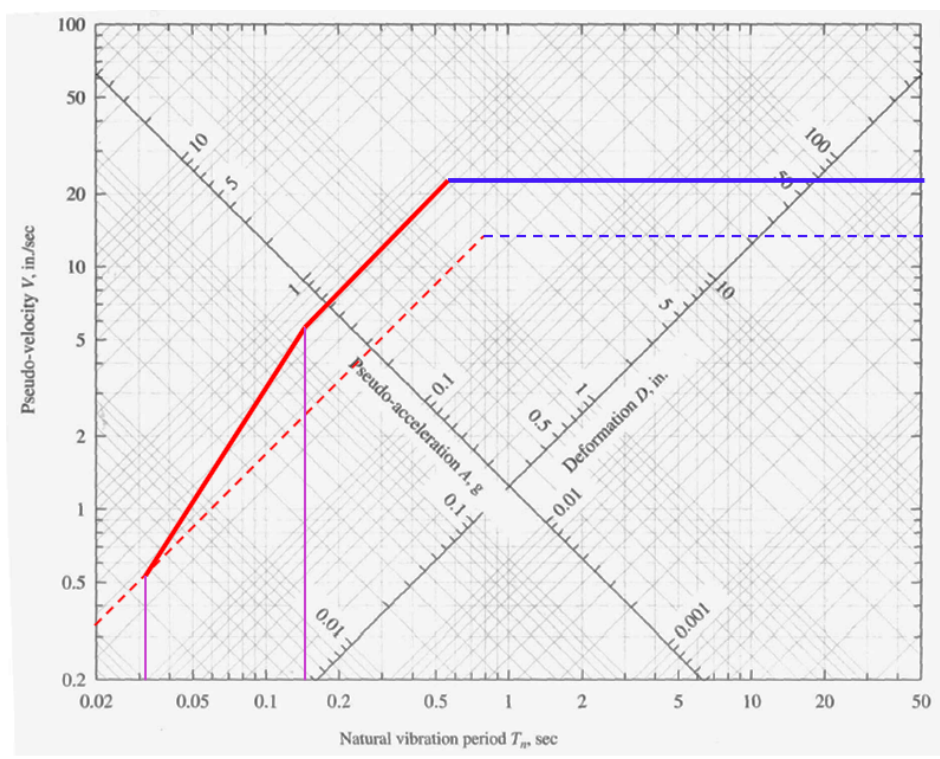

Figure EE - Design Spectrum with amplified regions

The solid lines represent the amplified values, while the magenta lines represent the transition points at approximately $0.035 \mathrm{sec}$ and $0.125 \mathrm{sec}$.

\subsubsection{Collecting Deformation Values}

Once these regions have been correctly plotted, the displacement values can be read from the graph. The frequencies from the structure are located along the $\mathrm{x}$-axis, and then followed vertically until intersection with the design spectrum. The deformation values can then be read from the deformation axis. These deformation values are tangible values - they represent the maximum displacement expected in an earthquake. The green 
lines represent the first and second natural vibrating periods for Wall A (0.199 seconds and 0.066 seconds, respectively) in Figure FF below.

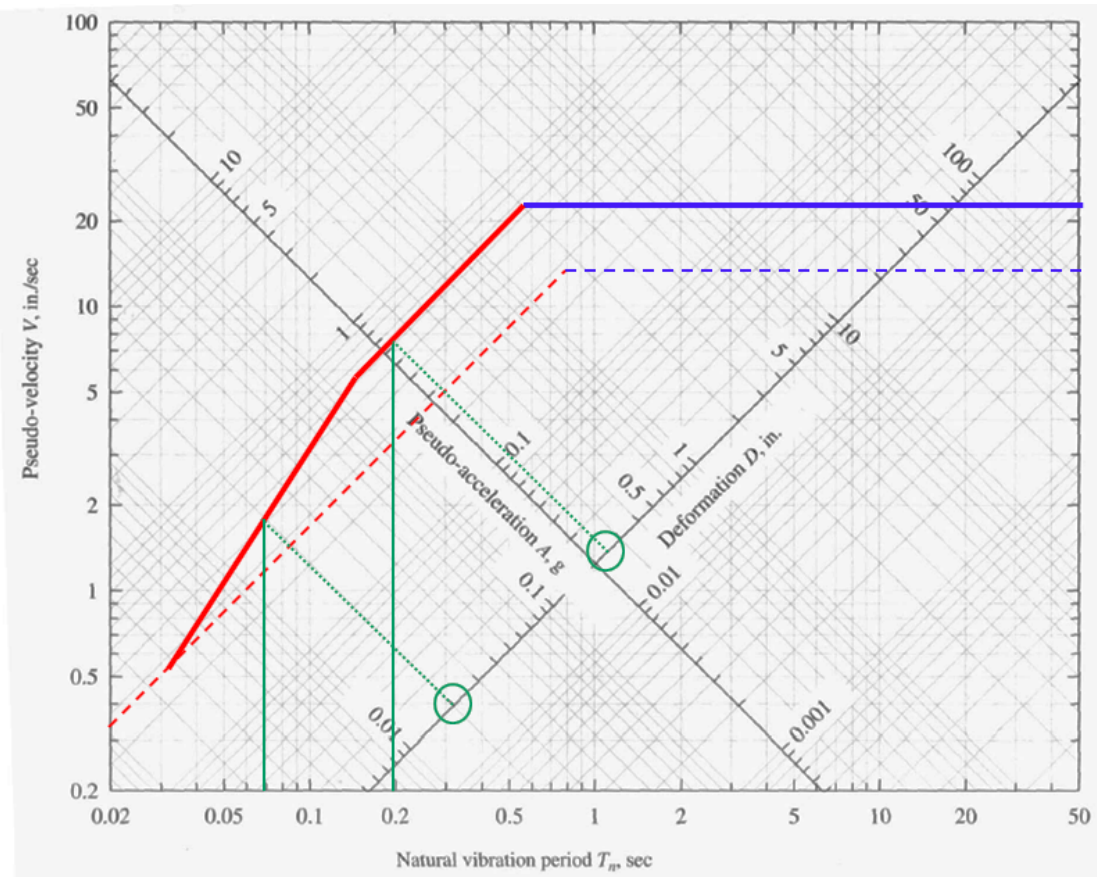

Figure FF - Design Spectrum with measured Deformation values

Each wall's unique design spectrum is created in the way described above, and each wall's deformation values are extracted accordingly. The deformation values (in inches) can be found listed below in Table 6 .

Table 6 - Spectral Deformation Values (inches)

\begin{tabular}{|c|c|c|c|c|c|}
\hline \multicolumn{2}{|c|}{ Wall A } & \multicolumn{2}{c|}{ Wall B } & \multicolumn{2}{c|}{ Wall C } \\
\hline Mode 1 & Mode 2 & Mode 1 & Mode 2 & Mode 1 & Mode 2 \\
\hline 0.180 & 0.015 & 0.170 & 0.012 & 0.350 & 0.015 \\
\hline
\end{tabular}




\subsection{ANALYSIS OF VIBRATION PROPERTIES}

Once the wall behavior and design spectrum information has been gathered, the two pieces can be joined for a complete modal analysis of the structural system.

\subsection{Formulation of Mass Matrices}

One of the first and most important steps in a modal analysis is defining the mass matrix. This definition of mass immediately presents multiple options in terms of how to accurately represent the mass. If the mass matrix is diagonal, that is, values only along the center diagonal of the matrix, then the assumption will be that only one section of the wall is excited at a time while in motion. If the mass matrix is tri-diagonal, or has 3 central diagonals in the matrix, then the assumption will be that the wall has a more distributed mass excitation. The only way to find which mass design will be the more accurate of the two is to do virtually the entire modal analysis with both mass matrices. In the end, the results will show which matrix form will be the most accurate application for this system.

For the lumped mass matrix, the weight is distributed per tributary interaction, as shown in Figure GG on the following page. 


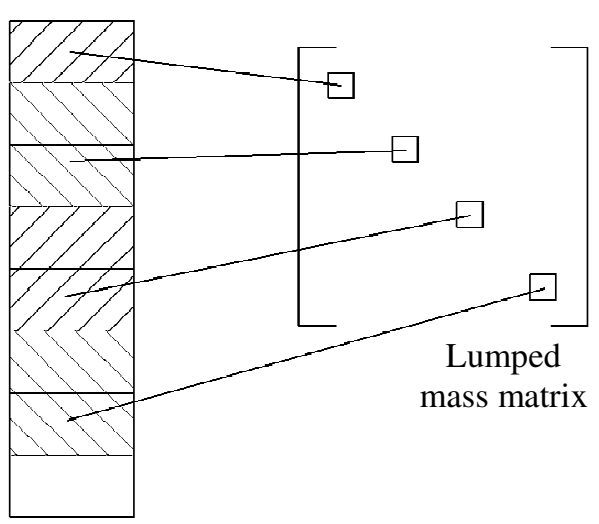

Figure GG - Summary of matrix formation

As seen in Figure GG above, the top diagonal value of the matrix has one-half the mass assigned to it, compared to the other three diagonal values. To calculate this mass value, the weight of one basket was divided by gravity, in units of in $/ \mathrm{sec}^{2}$. This principle comes from Newton's Second Law, $\mathrm{F}=\mathrm{ma}$, written similarly as $\mathrm{W}=\mathrm{m}^{*} \mathrm{~g}$. Thus, to get mass, the weight value must be divided by gravity. Since the PGV values are in units of in/sec, the units of gravity must also be in units of in $/ \mathrm{sec}^{2}$.

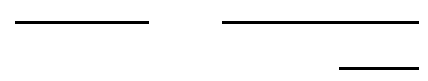

With each basket having a mass of $0.2047 \mathrm{lb}-\mathrm{sec}^{2} / \mathrm{in}$, or $0.2047 \mathrm{slugs}$, the diagonal mass matrix looks like the matrix below. 
The diagonal mass matrix is derived through the principle of virtual work, on the basis of linear shape functions. The shape functions for each degree of freedom considered in the structure can be seen in Figure GG below.

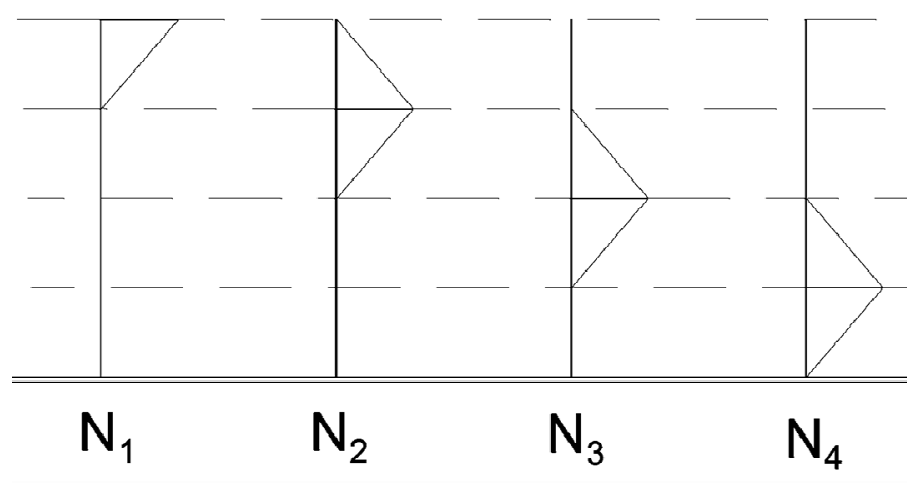

Figure HH - Shape Functions for Wall

Integrating these functions according to the form

results in the matrix and mass values below, as applied to this particular wall system.

$=$ 
As stated above, each of the calculations is done with both the diagonal mass

matrix and the tri-diagonal mass matrix. However, recognizing that the tri-diagonal mass representation is the more accurate assumption of the two, only the tri-diagonal results will be presented here.

\subsection{Ortho-Normalizing Modes}

After creating the mass matrix, each of the modes measured experimentally must be orthonormalized. The equation for this process can be found below (Chopra, 2007).

$$
\phi_{i}=\frac{\phi_{i}}{\sqrt{\phi_{i}^{T} * M * \phi_{i}}}
$$

where $\quad \square_{i}$ is the mode shape being orthonormalized, and

$M$ is the mass matrix for the structure under investigation.

By applying Equation 3 to each of the mode shapes presented in Mode Shapes (Section 4.1.4), the following orthonormalized modes are created. Again, these values are only the results that use the tri-diagonal mass matrix in the term $M$ due to the higher degree of accuracy from a more distributed mass matrix.

$$
\phi_{A}=\left[\begin{array}{cc}
2.519 & 3.946 \\
1.554 & -0.325 \\
0.800 & -1.526 \\
0.191 & -0.860
\end{array}\right] \quad \phi_{B}=\left[\begin{array}{cc}
2.446 & 3.583 \\
1.565 & -0.213 \\
0.790 & -1.725 \\
0.319 & -0.786
\end{array}\right] \quad \phi_{C}=\left[\begin{array}{cc}
1.880 & 3.740 \\
1.742 & -0.444 \\
0.886 & -1.480 \\
0.296 & -1.074
\end{array}\right]
$$

\subsection{Modal Sweeping}

The measured modes are not pure mode shapes, even after ortho-normalizing them. In shaking the walls at their measured natural frequencies, the hope is to excite the 
main respective mode while minimizing the other smaller modes. However, the other mode shapes will likely still be present. The only way to ensure that the collected data contains only the intended mode's behavior is to perform a modal "sweep". This is done by assuming one mode is "pure", and then "sweeping" another mode's behavior out of the results, a procedure termed the Modified Gramm-Schmidt (Golub, 1989) algorithm. The equation below guides this process.

$$
\phi_{2}^{\prime}=\phi_{2}-\frac{\left(\phi_{1}^{T} * M * \phi_{2}\right)}{\left(\phi_{1}^{T} * M * \phi_{1}\right)} * \phi_{1}
$$

where $\quad \square_{2}^{\prime}$ is the swept second mode,

$\square_{2}$ is the original second mode,

$\square_{l}$ is the original first mode which was assumed pure, and $M$ is the mass matrix.

This process results in the following values using the tri-diagonal mass matrix.

$\phi_{A}=\left[\begin{array}{cc}2.519 & 3.596 \\ 1.554 & -0.541 \\ 0.800 & -1.637 \\ 0.191 & -0.886\end{array}\right] \quad \phi_{B}=\left[\begin{array}{cc}2.446 & 3.415 \\ 1.565 & -0.321 \\ 0.790 & -1.780 \\ 0.319 & -0.808\end{array}\right] \quad \phi_{C}=\left[\begin{array}{cc}1.880 & 3.820 \\ 1.742 & -0.369 \\ 0.886 & -1.442 \\ 0.296 & -1.061\end{array}\right]$

After being swept, the modes need to be re-orthonormalized using the process laid out in Equation 4. Those values are found below.

$$
\phi_{A}=\left[\begin{array}{cc}
2.519 & 3.631 \\
1.554 & -0.547 \\
0.800 & -1.653 \\
0.191 & -0.885
\end{array}\right] \quad \phi_{B}=\left[\begin{array}{cc}
2.446 & 3.423 \\
1.565 & -0.322 \\
0.790 & -1.784 \\
0.319 & -0.810
\end{array}\right] \quad \phi_{C}=\left[\begin{array}{cc}
1.880 & 3.824 \\
1.742 & -0.369 \\
0.886 & -1.443 \\
0.296 & -1.062
\end{array}\right]
$$


The values seen before the modal sweep versus after the sweep do not show a great deal of difference. This lack of difference shows that the second mode is not largely dominated in behavior by the first mode, though a small amount of influence was swept out.

\subsection{Mass Participation Factors}

Once the mode shapes are swept and re-orthonormalized, they can then be used to calculate the mass participation factors (MPF). Through a series of equations, the MPFs basically show how much of the mass is excited in the modes being analyzed. For a typical structure, it is ideal to see at least $90 \%$ of the mass being excited in the first two modes. This is not an exact value, but rather an assumption that if $90 \%$ of the mass is captured in the modes of investigation, there is a basis for eliminating the need for higher-mode investigation (Thomson, 1981). The equation for the gamma value required to find the MPF is below.

$$
\Gamma=\Phi^{\mathrm{T}} * \mathrm{M} * \mathrm{~L}
$$

where $\Phi$ is the mode shape being analyzed,

$\mathrm{M}$ is the mass matrix, and

$\mathrm{L}$ is a unity vector, required to be taken to equal 1 . This occurs as representation that the base moves over 1 ; hence the entire structure moves 1 as well.

The gamma value is calculated for each mode individually, resulting in values found on the next page. 


$$
\begin{array}{ll}
\Gamma_{A-1}=0.686 & \Gamma_{A-2}=-0.355 \\
\Gamma_{B-1}=0.703 & \Gamma_{B-2}=-0.336 \\
\Gamma_{C-1}=0.717 & \Gamma_{C-2}=-0.291
\end{array}
$$

\subsubsection{Mass Participation}

On their own, the gamma values are not very useful. However, once squared and divided by the total mass, the gamma factors give the percentage of mass excited in the appropriate mode shape.

$$
\Gamma_{T O T}=\frac{\Gamma_{1}^{2}}{M_{t o t}}+\frac{\Gamma_{2}^{2}}{M_{t o t}}+\cdots
$$

This $\Gamma_{\text {TOT }}$ value is the overall percentage of mass excited in the included modes. For this experiment, only the first two modes are analyzed, so there are only two gamma terms to sum. The gamma terms seen above are squared, and then divided by the total mass of the structure, found by summing the mass matrix. These values can be seen for each of the walls below.

$$
\begin{aligned}
& M P F_{A}=[0.921] \\
& M P F_{B}=[0.937] \\
& M P F_{C}=[0.923]
\end{aligned}
$$

As previously discussed, the ideal MPF will be above 90\% (Thomson, 1981). This percentage shows that enough modes are analyzed to capture the vast majority of 
motion in the structure. Also, the results previously shown are for the tri-diagonal mass matrix, but for comparison, the MPFs for the diagonal mass matrix are shown below.

$$
\begin{aligned}
& M P F_{A}=[0.866] \\
& M P F_{B}=[0.896] \\
& M P F_{C}=[0.850]
\end{aligned}
$$

Since none of these values exceed $90 \%$, the diagonal mass matrix is shown to not be an accurate enough representation of the mass matrix. This result makes sense since the idea of only one section of the wall being excited at a time is not a realistic one.

\subsection{Expected Displacements}

Once the gamma values are found for each mode, they can then be used as scalar multipliers for the displacement values pulled earlier from the Design Spectrum. Those values have been restated here below for simplicity.

\begin{tabular}{|c|c|c|c|c|c|}
\hline \multicolumn{2}{|c|}{ Wall A } & \multicolumn{2}{c|}{ Wall B } & \multicolumn{2}{c|}{ Wall C } \\
\hline Mode 1 & Mode 2 & Mode 1 & Mode 2 & Mode 1 & Mode 2 \\
\hline 0.180 & 0.015 & 0.170 & 0.012 & 0.350 & 0.015 \\
\hline
\end{tabular}

Note that there is also one displacement value for each mode, just as with the gamma values. This is not a coincidence-each displacement value should be multiplied by its appropriate MPF to find its scaled displacement value. This product can then be multiplied by the mode shape itself to find what the actual displacements from the experiment would be. 
The equation for this calculation can be found below.

$$
d_{i}=\Gamma * D * \phi_{i}
$$

where $\quad d_{i}$ is the calculated displacement (inches),

$\Gamma$ is the gamma factor,

$D$ is the displacement value from the design spectrum, and

$\square_{i}$ is the swept, orthonormal mode shape.

The results for the tri-diagonal mass (Table 7) can be found below.

Table 7 - Summary of level displacements for each wall

\begin{tabular}{|c|c|}
\hline \multicolumn{2}{|c|}{ Wall A } \\
\hline Mode 1 & Mode 2 \\
\hline 0.0171 & -0.0006 \\
\hline 0.0402 & 0 \\
\hline 0.0208 & 0.0015 \\
\hline 0.0066 & 0.001 \\
\hline
\end{tabular}

\begin{tabular}{|c|c|}
\hline \multicolumn{2}{|c|}{ Wall B } \\
\hline Mode 1 & Mode 2 \\
\hline 0.0164 & -0.0004 \\
\hline 0.0387 & 0 \\
\hline 0.0206 & 0.0011 \\
\hline 0.0084 & 0.0007 \\
\hline
\end{tabular}

\begin{tabular}{|c|c|}
\hline \multicolumn{2}{|c|}{ Wall C } \\
\hline Mode 1 & Mode 2 \\
\hline 0.031 & -0.0005 \\
\hline 0.0833 & -0.0001 \\
\hline 0.0477 & 0.0011 \\
\hline 0.0177 & 0.0008 \\
\hline
\end{tabular}

Note that these displacement values (in inches) are for the individual levels of each of the walls and must be kept separate for the sake future calculations.

\subsubsection{Corresponding Forces}

Through a method described in Chapter 13 of Chopra's book (Chopra, 2007), these calculated displacements can be used to find the corresponding forces at each level. Knowing the forces is valuable for understanding how the structure is behaving towards the earthquake. Knowing the forces also allows additional mathematical analysis to further confirm or deny the usefulness of this particular structural system.

In Chopra's book (Chapter 13, Section 1), the following equation is prescribed for "backing out" forces once given the displacements (Chopra, 2007). 


\subsection{ANALYSIS OF VIBRATION PROPERTIES 56}

$$
f_{n}(t)=\omega_{n}^{2} * m * \phi_{n} * q_{n}(t)
$$

where $\quad \omega_{n}$ is the modal frequency converted into radians, $m$ is the mass, $\square_{n}$ is the swept and normalized mode shape, and $q_{n}(t)$ is the abbreviation for $D_{n} * \Gamma_{n}$, the displacement value from earlier.

Plugging all the appropriate values into Equation 6 above for each mode shape, the following forces are found—both for the individual modes and the combined forces.

Table 8 - Summary of forces for each wall (lbs.)

\begin{tabular}{|c|c|c|c|c|c|c|c|c|}
\hline \multicolumn{3}{|c|}{ Wall A } & \multicolumn{3}{c|}{ Wall B } & \multicolumn{3}{c|}{ Wall C } \\
\hline Mode 1 & Mode 2 & Total & Mode 1 & Mode 2 & Total & Mode 1 & Mode 2 & Total \\
\hline 22.75 & -7.20 & $\mathbf{2 3 . 8 6}$ & 13.66 & -3.89 & $\mathbf{1 4 . 2 0}$ & 27.05 & -5.89 & $\mathbf{2 7 . 6 9}$ \\
\hline 53.27 & 0.50 & $\mathbf{5 3 . 2 8}$ & 32.35 & -0.43 & $\mathbf{3 2 . 3 6}$ & 72.71 & -1.51 & $\mathbf{7 2 . 7 3}$ \\
\hline 27.63 & 18.80 & $\mathbf{3 3 . 4 2}$ & 17.18 & 10.39 & $\mathbf{2 0 . 0 8}$ & 41.70 & 12.29 & $\mathbf{4 3 . 4 7}$ \\
\hline 8.73 & 12.25 & $\mathbf{1 5 . 0 4}$ & 7.04 & 6.35 & $\mathbf{9 . 4 8}$ & 15.45 & 9.72 & $\mathbf{1 8 . 2 5}$ \\
\hline
\end{tabular}

\subsubsection{Free-body Analysis}

The forces found with Eq. 7 can now be used for further mathematical analysis. Simple as it may seem, a free-body diagram can be used to solve for the eccentricity of the soil's restoring force in reaction to the forces found above since the analysis assumes linear behavior. 


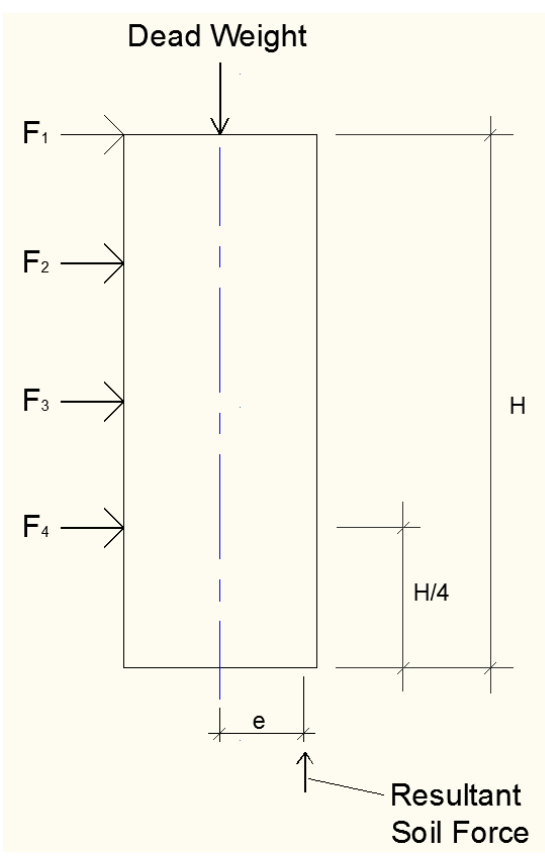

Figure II - Free body diagram for summation of moments

As seen in Figure $\mathrm{HH}$ above, the forces can be re-applied mathematically to the structure, and the occurring moments can be summed around the centerline of the structure. This summation essentially puts the soil reaction force against the resultant structure forces, since the moments are summed along the centerline of the wall, where the dead weight of the structure acts. In solving the simple summation of moments at the center of the wall base, the following eccentricities for the soil restoring force are calculated.

$$
e_{A}=4.7743^{\prime \prime} \quad \mathrm{e}_{\mathrm{B}}=3.8634^{\prime \prime} \quad e_{C}=7.1655^{\prime \prime}
$$




\subsection{Discussion of Results}

It should be restated that this system is assumed to be linear, so the estimate of $e$ is based on a linear structure using mode shapes and frequencies from small-amplitude displacement. Based on this assumption, the results from the summation of moments show the wall would not be standing after an earthquake. The wall width from the center of the wall to the edge is only $3^{\prime \prime}$, so to have an eccentricity over 3 " for each wall case means that the soil cannot resist the loads put on it by the structure. On paper, this would result in a failure for the wall and dismissal of the proposed idea.

Though this process works with small displacements, the damping ratios used for the design spectra reflected damping ratios gathered via large displacement behavior. Thus, we can determine maximum displacements from an earthquake using the created design spectra. With the linear assumption, the forces causing these displacements can be backed out to find the eccentricity of the restoring soil forces (this process is further investigated in Appendix A). The results from this process do not allow a conclusion for failure, but rather, a conclusion of non-linear behavior. Clearly, the wall softens at larger displacements—but it is quite possible that the structure does not fail. 


\subsection{CONCLUSION AND RECOMMENDATIONS}

When assumed to behave linearly, the analysis reports failure for the wall after only $1 / 2^{\prime \prime}$ of relative displacement. The major conclusion of this experiment, then, is that the system cannot be assumed to remain linear during an earthquake—at least those earthquakes used to construct the design spectra. Due to the lack of research in this area, this experiment can neither be deemed a success nor a failure. Rather, further research must be done into the nonlinear properties of a free-standing gabion wall in order to draw more definitive conclusions.

There are several parts of the testing that had to be compromised due to issues either with machinery or supplies. The ideal situation would have been to build a fullscale model on a shake table capable of putting out ground motions similar in magnitude to the expected seismicity levels of the proposed build region. Had the shake table been properly functioning, the response of the system due to ground motions could have been measured. As it was, the non-functioning table limits investigation of true amplitudedependent behavior, so there is no opportunity to confirm some of the results gathered analytically. Had the shake table been large enough, the system could have been built to full scale. Had enough lead been available, the mass of the system could have been entirely contained in the baskets, rendering the exterior weights unnecessary, and the fill of the baskets more homogenous. Even in the fill of the baskets, having non-homogenous shapes in the aggregate, steel plugs, and lead shot prohibited more accurate results.

Unfortunately, the testing conditions were not ideal, and thus left a great amount of room for future investigation in this area of experimentation. 
A more detailed investigation into the interior behavior of the gabion wall system may prove beneficial for this proposed structural type as well. For example, how does the interaction of the rocks used to fill the baskets affect the behavior of the over-all system? How does the soil interaction affect the structural behavior? How can the baskets be better modeled since there would be a large amount of rocking and subsequent energy absorption that occurs compared to a more rigid, wall-like approach? But most important, how does this wall behave when treated as the nonlinear system it so clearly is?

However, one wall design did turn out to be more successful than the other two: Wall B. The MPF was highest for Wall B (93.7\%) and the eccentricity for the restoring soil force was less than half of the other two. If further research is done on this particular topic, the cable design for Wall B can be taken as the most successful of the three cable designs presented in this experiment. All that can be accurately said is that this system is clearly nonlinear, and more research must be done in the light of this discovery for a more accurate view of true gabion behavior in seismic zones. 


\subsection{REFERENCES}

(Buckingham, 1915). Buckingham, E. "The Principle of Similitude.” Nature 99th ser. (1915): 396-97. Web.

(Chen, 2009). Chen, Grace Y. Analysis of Stabilized Adobe in Rural East Africa. Thesis. California Polytechnic University, San Luis Obispo, 2009.

(Chopra, 2007). Chopra, Anil K. "Free Vibration.” Dynamics of Structures: Theory and Applications to Earthquake Engineering. Upper Saddle River, NJ:

Pearson/Prentice Hall, 2007.

(Felix and Germain, 1996). Felix, Jean-Pierre, and Germain Goffin. "What is a Gabion?" Gabion Design. Web.

(Golub, 1989). Golub G.H. and Van Loan C.F. 1989. Matrix Computations: Second Edition, Johns Hopkins University Press, Baltimore.

(McDaniel and Archer, 2009). McDaniel, Cole C., and Graham Archer. "Real-Time, Full-Scale Building Dynamics Laboratory.” 2009.

(MGS, 2006). "Gabion Walls Design.” Erosion Control by Modular Gabion Systems. Modular Gabion Systems, 2006. Web.

(Newmark and Hall, 1982). Newmark, N. M., and W. J. Hall. Earthquake Spectra and Design. Berkeley, CA: Earthquake Engineering Research Institute, 1982.

(Shevchenko 1996). Shevcheko, K. I. "Gabions - Reliable Protection from Erosion" Hydrotechnical Construction 30-11 (1996). 676-680.

(Simac, et al. 1997). M. R. Simac, Bathurst, R.J., Fennessey, T. W. "Case study of a hybrid gabion basket geosynthetic reinforced soil wall" Ground Improvement 1 (1997) 9-17

(Thomson, 1981). W. Thomson, Theory of Vibration with Applications 2nd Edition, Prentice Hall, New Jersey, 1981.

(USGS, online). U.S. Geological Survey. Web. 9 Aug. 2010. Web.

(Zarnic, et al. 2001). Zarnic, Roko, Samo Gostic, Adam J. Crewe, and Colin A. Taylor. "Shaking Table Tests of 1:4 Reduced-scale Models of Masonry Infilled Reinforced Concrete Frame Buildings." Earthquake Engineering \& Structural Dynamics 30.6 (2001): 819-34. 


\section{APPENDIX A}

The experimental results from the modal analysis did not seem compatible compared to the displacement experienced and the corresponding force. The method for proving these results is to create an analytical model and send it through the same series of procedures as the experimental results.

\section{Formulation of Simplified Model}

Using a computer programming language called MATLAB ${ }^{\circledR}$, an artificial stiffness matrix and mass matrix was used to produce a set of corresponding mode shapes. These mode shapes then went through the modal analysis laid out in Analysis of Vibration Properties (Section 6.0), and the results were compared to the experimental results. In order to have semi-comparable results, the same tri-diagonal mass matrix from the experimental analysis was used. The stiffness matrix then needed to be designed for comparable results.

\section{Mass and Stiffness Matrix}

The same tri-diagonal stiffness matrix from the experimental procedure is used for the model modal analysis:

$$
M_{\text {tri-diag }}=\left[\begin{array}{cccc}
0.0341 & 0.0341 & 0 & 0 \\
0.0341 & 0.1365 & 0.0341 & 0 \\
0 & 0.0341 & 0.1365 & 0.0341 \\
0 & 0 & 0.0341 & 0.1365
\end{array}\right]
$$


In order to understand the formulation of the stiffness matrix, the program

MATLAB $®$ uses to produce these results must be touched on briefly (the technicalities of the language are beyond the scope of this thesis). Once the mass and stiffness matrix have been defined in the program, the following script can be entered into MATLAB to produce the mode shapes and frequencies:

$$
[\mathrm{phi}, \mathrm{lam}]=\operatorname{eig}(\mathrm{K}, \mathrm{M})
$$

where $\quad K$ is the defined stiffness matrix, $M$ is the defined mass matrix (here, tri-diagonal), eig is the function required to produce the mode shapes and frequencies, $p h i$ is the mode shapes returned in a matrix, and lam is a diagonal matrix containing the frequencies in units of $(\mathrm{rad} / \mathrm{sec})^{2}$.

Iterating through the MATLAB ${ }^{\circledR}$ program to find accurate properties for the model's results requires a basis for comparison. The values for mode shapes given by the "phi" matrix are unique to each structure type, so the stiffness cannot be tuned to match mode shapes. This left the frequency values given in the "lam" matrix. This matrix is also referred to as the "eigen value" matrix — thus, the "eig" short-name in the function. In tuning the natural frequencies of the computer model to those of the walls, the model is given the best chance of producing results that will be comparative to those gathered experimentally.

The "lam" matrix is set up in the matrix structure shown on the next page. 


$$
\operatorname{lam}=\left[\begin{array}{cccc}
\Omega_{1}^{2} & 0 & 0 & 0 \\
0 & \Omega_{2}^{2} & 0 & 0 \\
0 & 0 & \Omega_{3}^{2} & 0 \\
0 & 0 & 0 & \Omega_{4}^{2}
\end{array}\right]
$$

where $\quad \Omega$, or $\omega$, is the frequency of the structure converted to $\mathrm{rad} / \mathrm{sec}$.

It will be difficult to ensure that both the first and second frequencies are matched for both the computer model and the experimental wall, so the purpose for iteration becomes matching only the first natural frequency of an experimental wall.

Wall A is chosen to compare results to due to its less-extreme mode shapes. From Vibration Study's Discussion of Results (Section 4.1.5), Wall A's first natural frequency is $4.90 \mathrm{~Hz}$. The equation below shows the conversion from frequency to the units MATLAB ${ }^{\circledR}$ displays in its results.

$$
\Omega^{2}=\left[f_{n} * 2 \pi\right]^{2}=[4.90 * 2 \pi]^{2}=948(\mathrm{rad} / \mathrm{sec})^{2}
$$

where $f_{n}$ is the natural frequency of the structure, and $\Omega^{2}$ is the result given by MATLAB $®$, the eigen value.

The stiffness matrix then needs to be designed in such a way as to cause the first eigen value to be around $948(\mathrm{rad} / \mathrm{sec})^{2}$. The basic structure of a single degree of freedom (SDOF) system can be found below, the derivation of which is outside the scope of this thesis. 


$$
K=\left[\begin{array}{cccc}
k & -k & 0 & 0 \\
-k & 2 k & -k & 0 \\
0 & -k & 2 k & -k \\
0 & 0 & -k & 2 k
\end{array}\right] \text { lbs./in }
$$

The value " $\mathrm{k}$ " is then iterated through until the function $[\mathrm{phi}, \operatorname{lam}]=\operatorname{eig}(\mathrm{K}, \mathrm{M})$ produces the desired first frequency, approximately. In iteration, this value for ' $\mathrm{k}$ ' turns out to be $k=1187.0$. The final stiffness matrix for the computer model, then, looks like the one seen below.

$$
K=\left[\begin{array}{cccc}
1187 & -1187 & 0 & 0 \\
-1187 & 2374 & -1187 & 0 \\
0 & -1187 & 2374 & -1187 \\
0 & 0 & -1187 & 2374
\end{array}\right] \text { lbs./in }
$$

\section{Procedure for Comparative Analysis}

As stated earlier, the procedure used to process the mode shapes produced by MATLAB® is identical to that used for the experimental mode shapes. The first two mode shapes and frequencies put out by MATLAB® can be seen below.

$$
\begin{array}{cc}
\omega_{\mathrm{n}-1}=5.0 \mathrm{~Hz} & \omega_{\mathrm{n}-2}=15.9 \mathrm{~Hz} \\
\phi_{1}=\left[\begin{array}{c}
1.617 \\
1.527 \\
1.185 \\
0.646
\end{array}\right] & \phi_{2}=\left[\begin{array}{c}
1.780 \\
0.983 \\
-1.134 \\
-1.728
\end{array}\right]
\end{array}
$$

These mode shapes go through the same process as the experimental results:

1. Modes are orthonormalized with respect to the tri-diagonal mass matrix. 
2. Mode 1 is assumed pure, and Mode 2 was "swept" of Mode 1.

3. Mode 2 is re-orthonormalized after being "swept".

4. The gamma factor and corresponding MPF is found.

5. The design spectrum deformation values are found according to the natural vibrating periods.

6. The deformations are amplified and corresponding forces found.

7. The forces are entered into a FBD and the reactant soil eccentricities found.

The most relevant results for the experimental model begin at Step \#4. The MPF for the computer model is 0.9924 , or $99.2 \%$ mass excitation-an excellent sign for a proper modal analysis. The displacements and forces the model experienced are reported below.

Table 9- Displacements and forces for model results

\begin{tabular}{|c|c|}
\hline \multicolumn{2}{|c|}{ Displacements } \\
\hline Mode 1 & Mode 2 \\
\hline 0.02 & -0.0004 \\
\hline 0.0566 & -0.0007 \\
\hline 0.0439 & 0.0008 \\
\hline 0.0239 & 0.0012 \\
\hline
\end{tabular}

\begin{tabular}{|c|c|}
\hline \multicolumn{2}{|c|}{ Forces } \\
\hline Mode 1 & Mode 2 \\
\hline 30.7830 & 20.1392 \\
\hline 57.0638 & 56.3407 \\
\hline 45.1781 & 44.0835 \\
\hline 28.4326 & 26.4482 \\
\hline
\end{tabular}

Already, the same trend of small displacements, large forces can be seen in the computer results. For the sake of completion, the eccentricity of the soil restoring force is calculated and was found to be $6.92 "$ - well beyond the 3 " of width from the centerline of the wall to the outer extremity. 
Because the stiffness matrix is derived, this model procedure allows for another check that was not previously available with the experimental data. The inverse of that stiffness matrix can be multiplied by the force matrix, the resultant of which will give a deformation matrix. These values should be in the same approximate magnitude as the displacements used to back out the forces. In performing this calculation, the displacements are found to not exceed $0.3^{\prime \prime}$, so the model results are considered valid.

\section{Discussion of Model Results}

Ultimately, the purpose of the computer model is to create an ideal system through the designing of the stiffness and mass matrix. The results seen from the computer model are similar in pattern to those seen experimentally, especially in regards to the relationship between the experienced displacement and subsequent forces. If these results had not been similar in this way, the mode shapes gathered experimentally would have been deemed incorrect and another analytical mistake would have to be found. Because the results do mimic each other between the computer model and the experimental model, the analytical process is deemed correct and the resulting data judged to be accurate per experimentation. 